\title{
How cellular movement determines the collective force generated by the Dictyostelium discoideum slug
}

\author{
John C. Dallon ${ }^{\mathrm{a}, *}$, Hans G. Othmer ${ }^{\mathrm{b}, 1}$ \\ ${ }^{a}$ Department of Mathematics, Brigham Young University, 312 TMCB, Provo, UT 84602-6539, USA \\ ${ }^{\mathrm{b}}$ Max Planck Institute for Mathematics in the Sciences, Inselstrasse 22, Leipzig, Germany
}

Received 8 January 2004; received in revised form 28 April 2004; accepted 22 June 2004

Available online 3 August 2004

\begin{abstract}
How the collective motion of cells in a biological tissue originates in the behavior of a collection of individuals, each of which responds to the chemical and mechanical signals it receives from neighbors, is still poorly understood. Here we study this question for a particular system, the slug stage of the cellular slime mold Dictyostelium discoideum (Dd). We investigate how cells in the interior of a migrating slug can effectively transmit stress to the substrate and thereby contribute to the overall motive force. Theoretical analysis suggests necessary conditions on the behavior of individual cells, and computational results shed light on experimental results concerning the total force exerted by a migrating slug. The model predicts that only cells in contact with the substrate contribute to the translational motion of the slug. Since the model is not based specifically on the mechanical properties of Dd cells, the results suggest that this behavior will be found in many developing systems.
\end{abstract}

(C) 2004 Elsevier Ltd. All rights reserved.

Keywords: Dictyostelium discoideum; Cell movement; Motive force; Cell-based model; Visco-elastic elements; Migrating slug

\section{Introduction}

The individual and collective or tissue-like movement of cells is an important component of many processes, including morphogenesis and embryonic development, the immune response, wound healing, and angiogenesis and metastasis in cancer. In these processes individual cells detect chemical and mechanical signals in their environment and alter their pattern of movement in response. An example occurs in the chemotactic response of cells to attractants, which involves both a determination of the appropriate direction in which to move, and organization (or reorganization) of the motile machinery to initiate movement. In some instances cells

\footnotetext{
${ }^{*}$ Corresponding author. Tel.: +1-801-422-1205; fax: +1-801-4220504 .

E-mail address: dallon@math.byu.edu (J.C. Dallon).

${ }^{1}$ Present address: School of Mathematics, 270A Vincent Hall, University of Minnesota, Minneapolis, MN 55455, USA.
}

modify their environment by secreting signals, or by selectively degrading the matrix immediately surrounding them to facilitate movement or to amplify signals. In the immune response leukocytes migrate through the extracellular matrix as individuals, but in early embryonic development of vertebrates large-scale reorganization of cells occurs via a collective, tissue-like movement in which cells interact strongly.

Movement of the cellular slime mold Dictyostelium discoideum $(\mathrm{Dd})$ and other amoeboid cells involves at least four different stages: protrusion, attachment to the substrate, translocation of the cell body, and detachment of the rear (Mitchison and Carmer, 1996; Sheetz et al., 1999). (1) Cells first extend localized protrusions at the leading edge, which take the form of lamellipodia, filopodia or pseudopodia. Most current models explain force generation at the leading edge by localized actin polymerization and cross-linking (or gelation) of actin filaments. Behind the protrusion there is a region of actin disassembly, where filaments are disassembled, 
cross-links broken and the actin monomers resulting from disassembly freed to diffuse to the site of active polymerization (Alberts et al., 2002). (2) Not all protrusions are persistent, in that they must anchor to the substrate or to another cell in order for the remainder of the cell to follow (Soll, 1995). Protrusions are stabilized by formation of adhesive complexes, which serve as sites for molecular signaling and also transmit mechanical force to the substrate. In fibroblasts adhesive complexes are regions of the plasma membrane where integrin receptors, actin filaments and associated proteins cluster together. During migration the small nascent adhesive complexes (focal complexes) at the front of the cell grow and strengthen into larger, more organized focal adhesions that serve as traction 'pads' over which the cell body moves (Small, 1989). The molecular nature of the contacts is poorly characterized in $\mathrm{Dd}$, which does not express integrins, but one candidate molecule has recently been discovered (Fey et al., 2002). (3) Next, actomyosin filaments contract at the front of the cell and pull the cell body toward the protrusion in fibroblasts, whereas in $\mathrm{Dd}$, contraction is at the rear and the cytoplasm is squeezed forward. (4) Finally cells detach the adhesive contacts at the rear, allowing the tail of the cell to follow the main cell body.

Of course, to move cells must exert force on their surroundings, and how much force a cell exerts is also a function of the substrate: on a rigid, adhesive substrate they can generate large contractile forces transmitted through actin bundles and adhesion complexes, but on a pliable substrate they exhibit less organized actin and smaller, weaker adhesion complexes (Lo et al., 2000). In Dd the adhesive contacts are relatively weak and freeranging cells move rapidly $(\sim 20 \mu \mathrm{m} / \mathrm{min})$, whereas in fibroblasts they are very strong and cells move slowly.

\subsection{Models of cell and tissue movement}

Numerous models for the motion of individual cells on a substrate have been proposed. DiMilla et al. (1991) analysed a one-dimensional (1D) model in which a cell consisted of discrete subunits, each with an elastic spring, dash-pot and contractile element connected in parallel. Interaction with the substrate was modeled by additional Maxwell elements at the front and rear. The movement cycle was divided into three parts: the time needed for protrusion, the time of cytoskeleton contraction, and the time for cytoskeleton relaxation. Suitable choices of parameters lead to the 'bell-shaped' dependence of speed on adhesive properties of the substrate observed experimentally. A continuum model that incorporates further detail and leads to similar results was developed by Gracheva and Othmer (2003). A 1D model for the steady gliding movement of fish keratocytes was developed in Mogilner et al. (2000), where it was shown that the dynamics of self-alignment and contraction of the actin-myosin network can explain forward translocation of the cell body. (Mogilner and Verzi, 2002) developed a model for the crawling movement of nematode sperm consisting of a system of coupled differential equations that describe cell protrusion, contraction and adhesion. Under suitable biochemical regulation it produces steady motion of the cell. A two-phase flow model of cytoplasm designed to understand cytoplasmic streaming and oscillations was studied in Alt and Dembo (1999).

There are several other approaches to modeling cell deformations using a continuum description. The most widely used is the 'cortical shell-fluid core' model (Yeung and Evans, 1989; Skalak et al., 1990; SchimdSchönbein et al., 1995), in which the cortex is treated as a pre-stressed elastic medium and the core is treated as a fluid. This has been successful in explaining aspiration experiments (Yeung and Evans, 1989) and large, axisymmetric deformations of leukocytes (Dong and Skalak, 1992), but to date there is no 3D model based on this paradigm that incorporates active forces and arbitrary shape changes. ${ }^{2}$

However, to understand the collective tissue-like movement exhibited by cellular aggregates, one must understand how local interactions between moving cells affect the collective motion. In this paper we focus on the movement of Dd, and therefore we briefly review pertinent aspects of its life cycle.

In early aggregation the cells move autonomously, but in late aggregation they form connected streams that migrate toward the pacemaker. These streams converge in a loose mound of cells that usually topples over to form a cigar-shaped mass called the slug. The slug frequently moves in a pulsatile fashion by first elevating and extending the front and then pulling up the rear. At each stage, cells receive signals from the environment, and must integrate these signals, decide what direction to move, and organize their motile machinery to achieve this. A key issue is what force the slug exerts on a substrate and how this relates to the number of cells in the slug. Several early experimental studies by Inouye and Takeuchi $(1979,1980)$ and Inouye (1984) focused on the forces involved in slug movement, and their results will be discussed in detail later.

A cell-based model for motile Dd cells that can generate active forces, that interact via adhesive forces, and that can detect and relay chemotactic signals, was developed in Palsson and Othmer (2000). In the Palsson-Othmer (PO) model cells were treated as deformable viscoelastic ellipsoids, and signal transduction and cell-cell signaling was described using a previously developed model (Tang and Othmer, 1994,

\footnotetext{
${ }^{2} \mathrm{~A}$ recent step toward a continuum-based description of individual cells in which cells are treated as deformable spheres in epithelial layers is reported in Galle et al. (2004).
} 
1995; Dallon and Othmer, 1997, 1998). The shape constraint restricts the admissible deformations, but makes the simulation of thousands of interacting cells feasible. Since arbitrary ratios of semi-axes are allowed, subject only to the constraint of conserving the volume, the model admits a very wide range of shapes, from spherical to very long and slender. While single cells on rigid substrates are 'fried-egg'-shaped, cells in 3D matrices are more elliptical in shape (Wolf et al., 2003; Sahai and Marshall, 2003), and thus the shape restriction is not so severe in that context. This model can reproduce the observations on the chemotactic behavior of single cells, streaming during aggregation, and the collective motion of planar aggregates of cells driven by a small group of pacemakers. The model predicts that the motion of 2D slugs (Bonner, 1998) results from the same behaviors as are exhibited by individual cells; it is not necessary to invoke new mechanisms or behaviors.

In another cell-based model of $\mathrm{Dd}$, movement is governed by the minimization of a free energy (Savill and Hogeweg, 1997; Maree and Hogeweg, 2002). In this approach each cell is composed of several connected automata which change configuration according to prescribed rules, with the objective of minimizing the energy associated with bonds between cells. The objective was to model aggregation from single cells through the movement of a slug, and while the model can formally reproduce qualitative features of the aggregation and later stages, it is not clear how biochemical details about signal detection, rheological properties of the cytoplasm, and force generation can be incorporated into the rules of the model and related to energy minimization. For instance, active forces bias the energy function used in the minimization, but in reality these forces cannot be derived from a potential, and it is not clear whether this method can be made consistent with mechanical laws.

Other work has focused on continuum descriptions of either pattern formation in the Dd slug or on the movement of the slug. In the former category Pate and Othmer (1986) developed a model for scale-invariant patterning based on chemotaxis and cell sorting, and Umeda (1993) expanded on this work. In both cases the slug was represented as a continuum comprising prespore cells and pre-stalk cells at a constant total density. In Pate and Othmer (1986) chemotaxis equations were developed based on the differences of forces at the ends of a cell, while in Umeda (1993) an equation for the fraction of pre-stalk cells was formulated that incorporates adhesive differences that enter into a free energy which governs the evolution. The authors concluded that differences in chemotaxis was sufficient to cause sorting, and differential adhesion could create clear boundaries between the different cell types. Other continuum models that deal with the forces involved in movement of the slug will be discussed later.
Our objective here is to extend the PO model to make it applicable to 3D moving slugs by incorporating better descriptions of cell-cell and cell-substrate interactions, and to use the modified model to address some longstanding issues concerning the force exerted by a translocating slug. This involves several modifications in the way the forces are determined. For instance, in Palsson and Othmer (2000) net translocation of the center of mass of an aggregate required that all cells make contact with the substrate, but the $3 \mathrm{D}$ cell-sorting experiments done there do not require this. Here we remove this restriction, and the resulting model and software will provide a powerful tool for investigating many of the processes described above. Experimental data is now available which describes the mechanical behavior of individual cells, and it is desirable to include these details in a mathematical and computational model of individual cells. However, one must make simplifications if the objective is to study the interactions of many cells, and the major one in the PO model and here is that the cells are constrained to be ellipsoids. As we remarked earlier, results which show that the PO model can accurately represent the dynamics of $2 \mathrm{D}$ slugs suggest that the characterization of the interactions between cells is adequate and the shape constraint is not a severe limitation.

Here we focus on how cells in a Dd slug contribute to the overall motive force of the slug. In the following section we discuss some theoretical considerations that shed light on how cells can transmit stress to a substrate via other cells. We then describe in detail the properties of each cell, how they interact with each other, and how the boundary conditions are implemented in the PO model and how they are modified here. We also describe the algorithm for time stepping the equations of motion, and discuss the results of computational experiments aimed at understanding slug movement.

\section{Theoretical analysis of multicellular movement}

One difficulty in understanding the movement of the 3D slug in Dd is described by Odell and Bonner (1986), who say ".. in a close-packed 3D mass of cells, each amoeba trying to crawl can exert traction only upon its neighbors which, in turn, exert traction on it. In the interior of the grex (slug), with no rigid agar substratum to crawl upon, equal efforts by a cohort of amoebae to crawl in the same direction, each upon similarly crawling neighbors, cancel and produce no net mechanical result." One might expect that only the cells in contact with the rigid agar can move the slug forward, and that other cells are in effect carried by those cells in contact with the agar or substrate in a 'piggy-back' mechanism. On the other hand, some experiments suggest that the total motive force produced by the slug 
is proportional to its volume, and hence the total number of cells, which would rule out a 'piggy-back' mechanism (Inouye and Takeuchi, 1980; Inouye, 1984). As will be discussed in more detail later, many have simply postulated that the active motive force is a volumetric force in their macroscopic description of slug movement (Inouye and Takeuchi, 1979; Umeda and Inouye, 1999, 2002; Vasiev et al., 1997). Others have also proposed descriptions in which interior cells can transfer momentum to the substrate (Umeda and Inouye, 1999; Bretschneider et al., 1997, 1999) without a detailed analysis of how this occurs. Our first objective is to identify conditions under which a single-phase continuum model with suitable rheological properties derived from cell-level descriptions of movement will predict that the motive force is proportional to the volume of the slug. A comparison and critique of the Bonner-Odell model and other continuum models of the slug is given in Section 5.

Before describing a formal analysis of the problem, we can gain some insight into that analysis by the following heuristic argument. The question is what conditions in the interior of a slug would enable a cell to transmit force to the boundary and thereby to the substrate. In the absence of either action at a distance, or allowing cells to extend long filopodia to the substrate, it is clear that the force exerted by a cell has to be transmitted through other cells. However, if all cells are identical and attempt to transmit force simultaneously, Newton's third law shows that forces in the interior will cancel, which is the essence of the Bonner-Odell statement above. However, suppose that a portion of the cells are rigid and connected to other rigid cells in a chain that can transmit stress to the boundary. Then a motile cell in the interior can attach to a rigid cell and pull itself forward. We call this the bedspring model, since the rigid cells form a network that transmits stress to the boundary and allows other cells to crawl through it. This is similar to what occurs when a cell migrates through a tissue, but in order for mechanism to work in the Dd slug, the network must be dynamic and cells must be able to 'freeze' when stress is applied, but also to move. The following analysis formalizes this heuristic argument.

Suppose there are $N$ cells in the slug, and for simplicity let us frame the discussion in terms of axial forces that are oriented in the direction of motion so as to make the problem 1D. How cells select an orientation will be described in the following section. At present we do not stipulate that the total force on any cell, or on the slug itself, be zero, so there may be non-vanishing accelerations in the slug. Moreover we ignore deformations at present and regard all forces as acting at the center of mass. Deformation of cells and volume exclusion effects will be treated in detail later. The forces on a cell are those due to (i) the active forces it exerts on neighboring cells or the substrate, (ii) the reaction to forces exerted by other cells on it, (iii) dynamic drag forces that arise as a moving cell forms and breaks adhesive bonds with neighboring cells, and (iv) static frictional forces that exist when cells are rigidly attached to each other or to the substrate. The first three are self-explanatory and the latter will be defined precisely later. The active force cell $i$ exerts on the substrate or a neighboring cell is denoted $\mathbf{T}_{i, j}$, wherein $j=0$ denotes the substrate, and the reaction force to this is denoted $\mathbf{M}_{j, i}$ to indicate that this is the motive force. We have $\mathbf{M}_{j, i}=-\mathbf{T}_{i, j}$.

Let $\mathscr{N}_{i}^{a}$ denote the neighbors, including the substrate, of $i$ upon which it can exert traction. By definition, the 'neighbor' relation is symmetric for all cells; if cell $i$ can exert traction on cell $j$, then cell $j$ can exert traction on cell $i$, but this is not true for the substrate. The substrate is assumed to be passive, in that it does not generate stress. The total motive force generated by $i$ is

$\mathbf{M}_{i}=\sum_{j \in \mathscr{N}_{i}^{a}} \mathbf{M}_{j, i} \cdot$

Similarly, the total traction force which other cells exert on cell $i$ is

$\mathbf{T}_{i}=\sum_{j \in \mathscr{N}_{i}^{a}} \mathbf{T}_{j, i}$

In addition to traction forces, cells may exert drag forces on their neighbors as a result of their relative motion, and we define the dynamic friction force on cell $i$ due to interaction with $j$ as

$\mathbf{D}_{j, i}=\mu_{i j}\left(\mathbf{v} v_{j}-\mathbf{v}_{i}\right)$,

where $\mu_{i j}$ is the friction coefficient. This force satisfies the relationship $\mathbf{D}_{i, j}=-\mathbf{D}_{j, i}$, which implies that $\mu_{i j}=\mu_{j i}$. If $\mathbf{D}_{i}$ is the total dynamic friction force on cell $i$, and $\mathscr{N}_{i}^{d}$ is the set of cells that interact with $i$ via a frictional force, then

$\mathbf{D}_{i}=\sum_{j \in \mathscr{N}_{i}^{d}} \mathbf{D}_{j, i}=\sum_{j \in \mathcal{N}_{i}^{d}} \mu_{i j}\left(\mathbf{v}_{j}-\mathbf{v}_{i}\right)$.

Note that in general $\mathscr{N}_{i}^{d} \subseteq \mathscr{N}_{i}^{a}$ since cells may extend pseudopods to cells at some distance, but not be in contact with them in the sense of giving rise to a frictional force.

Finally, there is a static friction force on some cells, which is the means for constructing a network that can transmit stress to the boundary. This can be defined formally as a force that arises from infinitely tight binding between cells or from binding of a cell to the substrate. As a consequence of this binding, statically bound cells function as one rigid object. One might think of this force as a limiting case of the dynamic friction in which the friction coefficient goes to infinity, but a more precise description is that of the force between two 'welded' materials. Not all cells statically 
bind to other cells and we will use $\mathscr{N}_{i}^{s}$ to denote the set of cells that statically bind to cell $i$. This is a transitive relation in that if $i$ is bound to $j$ and $j$ is bound to $k$, then $i$ is bound to $k$. In particular, if $i$ is statically bound to the substrate either directly or indirectly then it can transmit forces applied to it to the substrate, thereby forming a component of the bedspring network. In fact these are the only type of chains that can transmit stress from the interior; a chain of say four cells statically bound to each other but not bound to the substrate simply functions as a larger unit. If $S_{j i}$ denotes the static binding force on the $i$-th cell when bound to the $j$-th, then $S_{j i}=-S_{i j}$ and the cell-cell forces cancel on all but those cells attached to the substrate.

The physical mechanism that mediates the static binding response and the adhesive interaction that enables a cell to generate active motive force is the same, but the underlying processes that initiate these are different. The active force arises when a cell initiates actin polymerization locally, extends a pseudopod, and anchors it on a neighboring cell via an adhesive complex. The target cell may then initiate the static frictional response in that it becomes rigid and binds very tightly to its neighbors. Of course the temporal sequence could be reversed, in that a cell extending a pseudopod only anchors to a cell that is statically attached to other cells. In reality the processes may occur simultaneously; contact of a pseudopod on a cell may initiate strain hardening in that cell and simultaneously lead to strengthening of the attachment via a complex feedback loop.

The total force on the $i$-th cell is

$$
\begin{aligned}
\mathbf{F}_{i} & =\mathbf{M}_{i}+\mathbf{T}_{i}+\mathbf{D}_{i}+\sum_{j \in \mathscr{N}_{i}^{s}} \mathbf{S}_{j i} \\
& =\sum_{j \in \mathscr{N}_{i}^{a}} \mathbf{M}_{j, i}+\sum_{j \in \mathscr{N}_{i}^{a}} \mathbf{T}_{j, i}+\sum_{j \in \mathscr{N}_{i}^{d}} \mu_{i j}\left(\mathbf{v}_{j}-\mathbf{v}_{i}\right)+\sum_{j \in \mathscr{N}_{i}^{s}} \mathbf{S}_{j i} .
\end{aligned}
$$

Some of these terms may be zero: for instance, $\mathbf{M}_{i}$ is zero for those cells that are not generating active motive force.

If we sum these over all cells we see that the sum of the tractions and motive forces, as well as sum of the dynamic friction force, vanish for all cell-cell interactions in the interior of the slug. The only surviving terms are those due to direct interaction of a cell with the substrate, or the indirect interaction via a chain of statically connected cells that is connected to the substrate. The latter takes the form of a force on an interior cell equal and opposite to the traction force it exerts on a statically connected chain of cells. Thus in the absence of static binding there is no mechanism by which an interior cell can transmit stress to the boundary, and accordingly, there can be no volumetric forces on the slug in a continuum description in the absence of other mechanisms. Bonner and Odell introduce additional mechanisms in the form of a gradient of cellular properties transverse to the direction of motion, but these have not be been confirmed experimentally.

A cell $i$ is said to be 'frozen' if it statically binds to the substrate directly or indirectly, that is, if there exists a sequence $j_{1}, j_{2}, \ldots, j_{k}$, such that $i$ statically binds to $j_{1}, j_{1}$ statically binds to $j_{2}, \ldots$, and $j_{k}$ statically binds to the substrate. The set of all frozen cells is called the frozen network of the slug. Thus the foregoing can be summarized as follows: in the absence of a frozen network in the slug, the total force on the substrate is the active force exerted by cells in direct contact with the substrate, and for a slug translocating at a constant velocity, this exactly balances the dynamic frictional interaction with the substrate.

\section{The computational model}

\subsection{An outline of the assumptions}

The PO model and the implementation of it that we describe here is based on two major assumptions: the cells are oriented ellipsoids and the passive response of the cytoplasm is that of an incompressible viscoelastic solid. In addition, there are assumptions as to how cells interact, how they orient to stimuli, and how active forces are exerted on neighboring cells. The shape constraint restricts the allowable deformations and in doing so makes possible the simulation of large populations of interacting cells. As remarked earlier, this constraint is not so important for cells in a 3D matrix, but it is less clear how important it is when cells are cheek-by-jowl and interact strongly. Work on a cellbased model that is free of this constraint is currently in progress. Since cells are assumed to be ellipsoids, nonspherical cells are assumed to reorient their major axis to coincide with the stimulus direction before translocation begins, which is a mathematical reflection of the biological observation that cells that have received stimuli tend to be polarized, thus have an orientation (see Fig. 1), and absent restraints from neighboring cells move in the direction of their orientation. The three major differences between the present implementation and that in Palsson and Othmer (2000) are the way in which the forces are determined, how the shape of the cell is calculated, and the interaction of cells with a fixed substrate. In addition to these major changes there are several implementational details, including the way in which the cells reorient and the numerical algorithms used for solving the equations of motion.

Directed cell motion in amoeboid cells is a complex process that can be separated into two independent subprocesses: the determination of a direction in which 


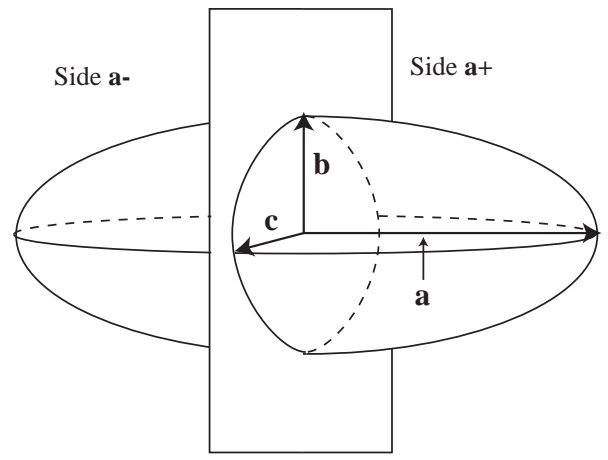

Fig. 1. The figure shows a schematic of a cell and the nomenclature used in the simulations. The labeled vectors $\mathbf{a}, \mathbf{b}$, and $\mathbf{c}$ indicate the major axes of the ellipsoid. The cell is oriented in the direction of vector a which corresponds with the direction of motion. Each crosssectional plane ab, ac or be separates the cell into a plus and minus sides with respect to the normal of the plane. For example the bc plane shown separates the cell into a plus and minus with respect to the a direction denoted $a+$ and $a-$, respectively.

to move, which we call orientation, and the motion itself, which involves the conversion of chemical energy into mechanical energy. The former involves a complex signal transduction pathway, the main steps of which are used in numerous amoeboid cells (Chung et al., 2001). Here we are not concerned with how the cell determines a direction or how it decides when to move, but only with the motion itself. Thus the model has two different inputs corresponding to the two processes: an orientation stimulus and a motion stimulus. The orientation stimulus provides a direction of orientation $\hat{\mathbf{d}}_{o}$, which is the direction the cell will turn towards as it starts to move and then it will continue moving in that direction. The motion stimulus determines one of two states for the motion, high motility or low motility. In the case of Dd, both inputs are derived from the cAMP signal. For the simulations in this paper low motility motion means no motion and therefore we do not distinguish between the terms high-motility motion or active motion.

The process of orienting a cell in the model is called an orientation cycle, and this consists of an ordered sequence of steps. The direction of motion may either be chosen randomly, if there is no directional signal, or it may be determined by the signal. Thus when cell motion is initiated, a cell will always start an orientation cycle, but a cell can continue to move after an orientation cycle has completed. The orientation cycle lasts a predetermined length of time, $t_{c}$ (for most simulations $20 \mathrm{~s}$ ). The orientation stimulus is considered only at the beginning of an orientation cycle and is then ignored until a new cycle is started. If an orientation cycle has been started and is not complete but the motion stimulus indicates high motility motion should stop, the orientation cycle will be terminated as will the motion. When high motility motion is terminated a new orientation cycle is initiated and low motility motion is started. These rules are chosen for two reasons. First, in previous modeling of Dd it was found that if a cell follows the gradient of the chemoattractant too closely, it will get lost in local peaks of the chemoattractant and cells cannot aggregate successfully (Dallon and Othmer, 1997). However the modeling also indicates that a successful strategy for aggregating is to maintain the same direction for a period of time and only change directions occasionally. Thus in the model cells do not constantly alter their course, but only use the direction stimulus at the beginning of an orientation cycle. Yet once a direction is chosen motion can persist for as long as the motion stimulus indicates. Second, experimental evidence indicates that Dd cells can alter their direction within $20 \mathrm{~s}$ after the location of a source of cAMP is changed (Futrelle et al., 1982).

After determining if a cell is in an orientation cycle or not, the forces acting on the cell are used to determine its motion. Three types of interactions are considered in the model and they give rise to the four types of forces described in Section 2. The three types of interactions are: cell adhesion, which gives rise to dynamic drag forces denoted $\mathbf{D}$ and static frictional forces denoted $\mathbf{A}$; active cell motion, which generates active forces giving the cell traction denoted $\mathbf{T}$; and interactions due to the physical presence of other cells (or the substrate) including their viscoelastic nature, which are denoted $\mathbf{R}$ and $\mathbf{R}^{*}$ (rheological forces), and the reactive force to tractional forces $\mathbf{M}$ (motive forces). In addition to these forces, the model also takes into consideration drag due to the surrounding fluid. A detailed discussion of how these forces are calculated is contained in Appendix A.

When determining how the forces affect a cell, the forces are resolved into the coordinate vectors defined by the three major axes of the cell. In addition, the forces in each of these coordinate axes act either on the plus side of the cell with respect to an axis or on the minus side of the cell with respect to an axis (see Fig. 1).

\subsection{The rheological model of the cytoplasm}

The cytoplasm in many amoeboid cells has been characterized as a viscoelastic material whose properties are dominated by actin filaments and microtubules, collectively termed the cytoskeleton (Janmey, 1991). Actin filaments are organized either as a meshwork in the leading edge, or as transient bundles of actin filaments that extend along the length of cells. The elastic modulus of actin solutions is concentration dependent (MacKintosh, 1998), and they exhibit strain hardening (Xu et al., 2000), a property that may be important in slug movement. Some even characterize the cytoplasm in Dd as an 'active viscoplastic' material (Feneberg and Westphal, 2001), because it exhibits 


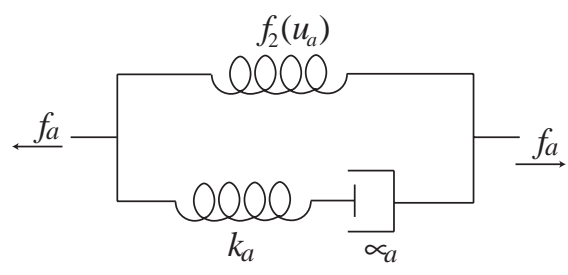

Fig. 2. A schematic of the element of a standard solid, or Kelvin element along each axis of the ellipsoid. It consists of a nonlinear spring in parallel with a Maxwell element, which comprises a linear spring in series with a dashpot.

viscoelastic behavior above a yield stress, but little deformation below this level.

The details of how actin networks are reorganized under the membrane in response to extracellular signals is a complex process that is only partially understood, and it is not feasible to incorporate the level of detail required to describe this in a multicellular model at present. In the PO model and here the opposite extreme in descriptions is adopted; cells are treated as incompressible viscoelastic solids and their stress-strain response characteristics are lumped into three elements that coincide with the three major axes. Along each axis the stress-strain behavior is described by a nonlinear version of a standard Kelvin element, as shown in Fig. 2.

Since there is an elastic element in each branch the immediate response to a step change in the applied force is a step change in displacement in the elastic elements, followed by slower flow in the viscous element. Release of the external forces leads to a return to the rest length of the element, and since we assume that the elements are identical along the three axes, the equilibrium shape is spherical.

However, the three deformations are not arbitrary, because the total volume of the cell must be conserved. ${ }^{3}$ In Palsson and Othmer (2000) the constant volume constraint was satisfied by first computing the deformation and then correcting the change in all radii so as to correct the volume. Here we adopt a method more in line with a continuum description of the material. Suppose that we write the Cauchy stress for a material in the form

$\mathbf{T}=-p \boldsymbol{\delta}+\tau$,

where $p$ is the mean isotropic stress and $\tau$ is the viscous stress tensor, which we assume is traceless. Suppose, for illustrative purposes, that the cytoplasm is a linear viscoelastic fluid, and thus assume in analogy with the Maxwell mechanical element that the rate of

\footnotetext{
${ }^{3}$ In reality this is probably not strictly correct, since osmotic fluxes may well change the total volume during deformations. However this has not been characterized experimentally, and incorporating it would add a great deal of complexity for an as yet unquantifiable increase in accuracy.
}

deformation tensor

$\mathscr{D} \equiv \frac{1}{2}\left(\nabla \mathbf{v}+(\nabla \mathbf{v})^{\mathrm{T}}\right)$

comprises an elastic part and a viscous part. That is, assume that the constitutive relation for the traceless part of the elastic component is

$\check{\tau}=k \mathscr{D}^{\circ}$

and for the viscous element

$\tau=\mu \mathscr{D}^{0}$,

where ${ }^{o}$ denotes the traceless part of a tensor and denotes an invariant time derivative, such as the Lie or the Jaumann derivative. Then

$\frac{\check{\tau}}{k}+\frac{\tau}{\mu}=2 \mathscr{D}^{\circ}$.

For the isotropic part we assume a similar decomposition and thus write

$\kappa \dot{p}+\frac{p}{\lambda}=-\operatorname{tr}(\mathscr{D})$,

where $\kappa \equiv \partial \ln V / \partial p$ is the compressibility, $\lambda$ is the bulk viscosity, and $\operatorname{tr}(\cdot)$ denotes the trace of a tensor. If the fluid is nearly incompressible $\kappa$ is small, and the pressure in a viscoelastic fluid equilibrates rapidly with the trace of the deformation rate. In the limit this is a singularly perturbed equation and sets a constraint on the deformation.

In the same way, we can decompose the total stress on an ellipsoid into the shear along the axes of the ellipsoid and an additional 'isotropic' part reflected in a pressure that constrains the overall deformation so as to satisfy the constraint that the volume remain constant. The rheological model we adopt is more complex than the Maxwell model used for illustration, but the same principle applies. In what follows the pressure is the deviation from a rest state and therefore may have either sign: a negative pressure causes the cell to compress and a positive pressure causes the cell to expand. In reality the cell must also be involved in setting the pressure, but the present formulation allows us to deform the cell as a viscoelastic substance and maintain a constant volume without modeling the complicated cell membrane.

The equation governing the length of an axis of a cell is

$$
\begin{aligned}
u_{a}^{\prime}= & \left(\frac{k_{a}}{\mu_{a}}\left[f_{a}(t)+\bar{p}-f_{2}\left(u_{a}\right)\right]+f_{a}^{\prime}(t)\right) \\
& \times\left(\frac{\mathrm{d} f_{2}\left(u_{a}\right)}{\mathrm{d} u_{a}}+k_{a}\right)^{-1},
\end{aligned}
$$

where $u_{a}$ is the change in the length of the a axis, $f_{2}$ is the force from the spring in parallel, $\mu_{a}$ is the viscous coefficient of the dashpot, $k_{a}$ is the spring constant for the spring in the Maxwell element, $f_{a}$ is the magnitude of the force applied at each end in opposite directions, 
and $\bar{p}$ is the force due to pressure. There are three such equations one for each axis and one equation due to the volume constraint given by

$$
\begin{aligned}
0= & u_{a}^{\prime}\left(u_{b}+b_{0}\right)\left(u_{c}+c_{0}\right)+\left(u_{a}+a_{0}\right) u_{b}^{\prime}\left(u_{c}+c_{0}\right) \\
& +\left(u_{a}+a_{0}\right)\left(u_{b}+b_{0}\right) u_{c}^{\prime} .
\end{aligned}
$$

where $a_{0}, b_{0}$, and $c_{0}$ are the initial lengths of the cell axes. This system of ordinary differential equations determines the shape of a cell and is solved using the software package dlsode.f (Radhakrishnan and Hindmarsh, 1994) at each time step.

Depending on the external forces acting on a cell, it will move, change shape or both. Consider the external forces acting on a cell parallel to the â axis. Let $f_{a+}$ be the sum of the magnitude of the forces acting on the side $a+$ and $f_{a-}$ be the sum of the magnitude of the forces acting on the side $a-$. The net force translates the cell and the change in shape in the direction $\mathbf{a}$ is determined by $f_{a}(t)$ in Eq. (3) which is given by

$f_{a}(t)=\left(\frac{f_{a+}}{\left|f_{a+}\right|}\right)\left(\frac{\left|f_{a+}\right|+\left|f_{a-}\right|-\left|f_{a+}+f_{a-}\right|}{2}\right)$.

\subsection{The equations of motion}

The equation of motion for the $i$-th cell can be written as

$$
\begin{aligned}
& \frac{A_{i f} \mu_{f}}{A \mu_{\text {cell }}} \mathbf{v}_{i}+\frac{A_{i s} \mu_{s}}{A \mu_{\text {cell }}} \mathbf{v}_{i}+\sum_{j \neq i} \frac{A_{i j}}{A}\left(\mathbf{v}_{i}-\mathbf{v}_{j}\right) \\
& \quad+\frac{1}{6 \pi r_{i b} \mu_{\text {cell }}}\left(\mathbf{M}_{0, i}+\mathbf{R}_{0, i}^{*}+\sum_{j \neq i} \mathbf{T}_{j, i}+\sum_{j \neq i} \mathbf{A}_{j, i}\right. \\
& \left.\quad+\sum_{j \neq i} \mathbf{R}_{j, i}+\sum_{j \neq i}\left(\mathbf{M}_{j, i}+\mathbf{R}_{j, i}^{*}\right)\right)=0 .
\end{aligned}
$$

As before the first subscript indicates the source and the second indicates the target of a force. Recall the subscript 0 denotes the substrate, $A$ denotes the total area of an undeformed cell, and $A_{i j}, A_{i f}$, and $A_{i s}$ denote the contact, area between cell $i$ and cell $j$, cell $i$ and the fluid, and cell $i$ and the substrate respectively. The parameters $\mu_{\text {cell }}, \mu_{s}$, and $\mu_{f}$ are determined by the degree of adhesiveness between cells, between the substrate and cells, and the fluid viscosity. Finally, $r_{i b}=u_{b}+b_{0}$ for cell $i$, and $\mathbf{v}_{i}$ denotes the velocity of cell $i$. The terms depending on velocity are drag forces, and because the Reynolds number for this system is very small we ignore the acceleration (see Appendix A for further details).

The system is solved using two different methods. The first is a quasi-implicit method based on the trapezoidal rule and the second is an Adams fourth-order predictor-corrector method. The final results of simulations using both methods compare well, but the quasi-implicit method requires more computational time. Thus for all the simulations shown in this paper, the predictor-corrector method was used. For more details on the quasiimplicit numerical method see Appendix A.

\subsection{Parameters}

There are several important parameters necessary for our model. It is possible to obtain estimates for several of these and in this section we discuss how the values used in the simulations were determined. Values of forces for several types of cells are given in Table 1. For typical simulations we use a high motility motion force of $4 \times 10^{-3}$ dyn.

The viscoelastic properties of leukocytes have been measured and we use those values as a guide to determine the spring constants and the dasphot constant for the viscoelastic elements in our model.

Table 1

Measured active forces and cell speeds

\begin{tabular}{lllr}
\hline Cell type & Active force & Source & Average speed $(\mu \mathrm{m} / \mathrm{min})$ \\
\hline Fibroblasts & $1 \times 10^{-2} \mathrm{dyn}$ & Harris et al. (1980) & 1 \\
Fibroblasts & $1.4-1 \times 10^{-1} \mathrm{dyn}$ & Dembo and Wang (1999) & 1 \\
Keratocytes & $2 \times 10^{-3} \mathrm{dyn}$ & Oliver et al. (1999) & 10 \\
Leukocytes & $3 \times 10^{-3} \mathrm{dyn}$ & Usami et al. (1992) & 20
\end{tabular}

Table 2

Viscoelastic parameters

\begin{tabular}{lclll}
\hline Cell type & $k_{2}\left(\mathrm{dyn} / \mathrm{cm}^{2}\right)$ & $k_{a}\left(\mathrm{dyn} / \mathrm{cm}^{2}\right)$ & $\mu_{a}\left(\mathrm{dyn} \mathrm{s} / \mathrm{cm}^{2}\right)$ & Source \\
\hline Leukocytes & 121.2 & 293 & 223.8 & Schmid-Schönbein et al. (1981) \\
Leukocytes & 7.5 & 238 & 330 & Sung et al. (1988) \\
Fibroblast & $500-1217$ & $1200-4000$ & $700-3000$ & Ward and Li (1991) \\
\hline
\end{tabular}


The observations from experiments on leukocyte deformations were fitted to a standard linear solid model of a cell (Sung et al., 1988). The parameters found are given in Table 2.

The values given are for a passive cell and slight displacements; they do not account for active deformation of a moving cell, which is important here. By examining the creep function and the relaxation function of a standard linear solid (see Appendix A) and considering the values of the above table, we determined the following parameter set which is used for a typical simulation: $k_{a}=k_{b}=k_{c}=$ $163.8 \mathrm{dyn} / \mathrm{cm}, k_{2}=147.5 \mathrm{dyn} / \mathrm{cm}$, and $\mu_{a}=\mu_{b}=\mu_{c}=$ $123 \mathrm{dyn} \mathrm{min} / \mathrm{cm}$. With these parameter a force of $400 \mathrm{nN}$ will deform the cell about $2.5 \mu \mathrm{m}$ in $4 \mathrm{~min}$ and it will return to its original shape $7 \mathrm{~min}$ after all forces are removed (see the appendix). The results of the paper are not sensitive to these parameter values, for example simulations have been run with $k_{2}$ ranging from 1.475 (making the cell much less stiff) to 1475.0 (making the cell even stiffer) and the same force relationship holds. If the parameters are chosen so that the cell is too stiff, numerical difficulties do arise. (Note the viscoelastic elements in the model are 1D, whereas the parameters in the table come from a constitutive equation for the standard solid model in two dimension.)

When the cell is compressed sufficiently it becomes much stiffer due to the different response of the nucleus (Caille et al., 2002), and to capture this we assume that $f_{2}$ is given as follows:

$f_{2}(x)= \begin{cases}k_{2} x, & x>-0.6, \\ -0.3 k_{2}\left(\frac{x}{0.3}\right)^{2}, & x \leqslant-0.6,\end{cases}$

where $x$ is the dimensionless displacement from 1 of the diameter of the cell. The value -0.6 corresponds to a cell nucleus with a diameter of $4 \mu \mathrm{m}$.

The glycoprotein csA is an adhesion molecule in Dd whose force in a cell-cell contact was measured by single-molecule force spectroscopy (Beniot et al., 2000), and we use the result to estimate the necessary adhesion parameters. The unbinding force was determined to be approximately $23 \mathrm{pN}$, and csA covers roughly $2 \%$ of the total cell-surface area at the aggregation stage (Beniot et al., 2000). It forms clusters of diameters from 30-70 nm (Harris et al., 2001), and assuming the cells are initially spherical and the diameter of the clusters is $40 \mathrm{~nm}$, we estimate $1.6 \times 10^{7}$ clusters $/ \mathrm{mm}^{2}$. Because our simulations are of the slug stage where the adhesion is higher, typical simulations use $3.2 \times 10^{8}$ clusters $/ \mathrm{mm}^{2}$ as the density of adhesion clusters, with $15 \mathrm{pN}$ of force per cluster. The contact area between two cells is determined in the simulations allowing the forces due to adhesion to be calculated.

The turning time, which in the model is the time it takes a cell to reorient to a new direction, is set at $20 \mathrm{~s}$.
This is taken from experiments which show cells can reorient to stimuli within $20 \mathrm{~s}$ (Futrelle et al., 1982). The coefficients $\mu_{s}, \mu_{\text {cell }}$, and $\mu_{f}$ are 27.0,27.0, and $2.7 \mathrm{dyn} \mathrm{s} /$ $\mathrm{cm}$, respectively.

\section{Simulations examining translational forces}

In this section we describe the results of several simulations designed to gain a better understanding of the translational force exerted by a moving slug. In the first set of experiments the starting configuration of the cells is that of a square slug. We simulate cases where all the cells move and where only a subset of the cells move. In the second set of simulations, the slug size varies in all dimensions resulting in some non-slug-like configurations. We will interpret these results in the context of the biological experiments which have been performed in Section 5 .

In the first set of simulations, the 'slug' initially has a square cross-section in the direction transverse to the motion. Only a band of cells transverse to the direction of motion are stimulated to move, in order to simulate the signal and response in a wave of cAMP. In these simulations about $20 \%$ of the cells are moving at any given time, but we also ran simulations in which all cells are moving. One can expect that these two simulations bracket the naturally occurring behavior in a slug, which ranges from a peristaltic motion to a smooth flow.

The starting configuration of the cells for 15 simulations is a slug 10 cells long with different-sized crosssectional squares ranging from 1 cell $\times 1$ cell to 15 cell $\times$ 15 cell. Fig. 3 shows results from a typical simulation at three different times.

The net motive force in the direction of motion can be determined by projecting the total force onto the direction of travel, subtracting the drag terms, and ignoring the scaling factor. This is calculated at each time step and is averaged over the last $8 \mathrm{~min}$ of each simulation. Fig. 4 shows the results plotted against the number of cells in contact with the surface and the total number of cells. In the model the volume is proportional to the total number of cells and the number of cells in contact with the surface is also proportional to the surface area in contact with the substrate.

The results in Fig. 4 clearly show that the force does not scale linearly with volume (or number; cf. (b)), as was concluded from experimental measurements (Inouye, 1984), but rather that it scales linearly with the contact surface area. Of course both plots show this, since they are based on the same data. In Fig. 4(b) the length is fixed at 10 cells, the number in a cross-section is essentially $N_{0}^{2}$ for variable $N_{0}$, the total number of cells is $N_{T}=10 N_{0}^{2}$, and the number in contact with the substrate is approximately $10 N_{0}=\sqrt{10 N_{T}}$. The 


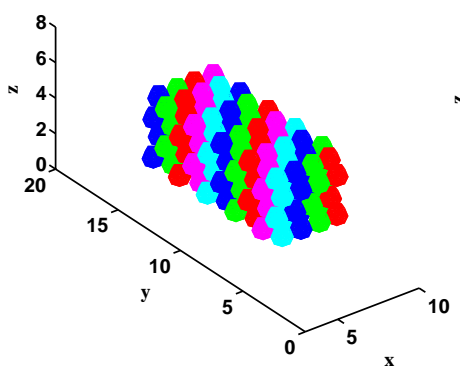

$\mathrm{a}: t=0$

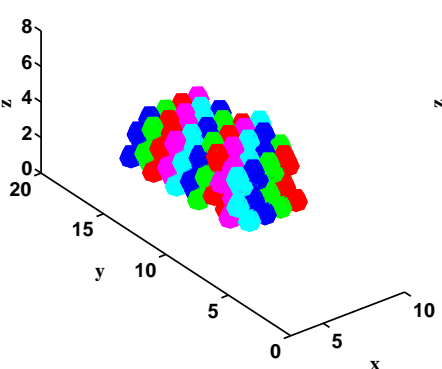

$\mathrm{b}: t=5 \mathrm{~min}$

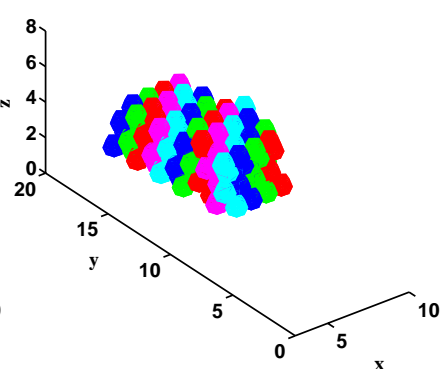

$\mathrm{c}: t=10 \mathrm{~min}$

Fig. 3. The results of a typical simulation are shown at three different times. At time $t=0$ the slug starts out 10 cells long with a square cross-section four cells on a side. A unit on the scale indicated corresponds to $10 \mu \mathrm{m}$. The only cells that move are those being simulated by a traveling wave, which is indicated in the foreground by the squares. The wave has a velocity of $30 \mu \mathrm{m} / \mathrm{min}$, a spatial period of $100 \mu \mathrm{m}$ and a width of $20 \mu \mathrm{m}$ in which the cAMP is non-zero. The spatial scale of the stimulus wave is smaller than in a natural wave to accommodate the small size of the slug.

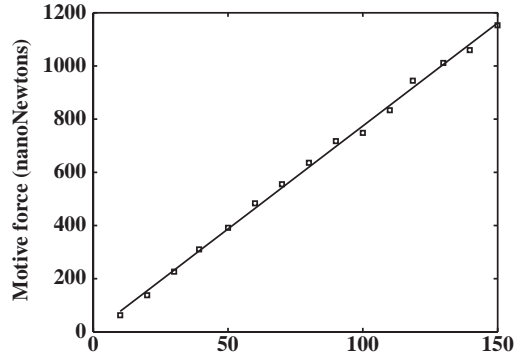

(a)

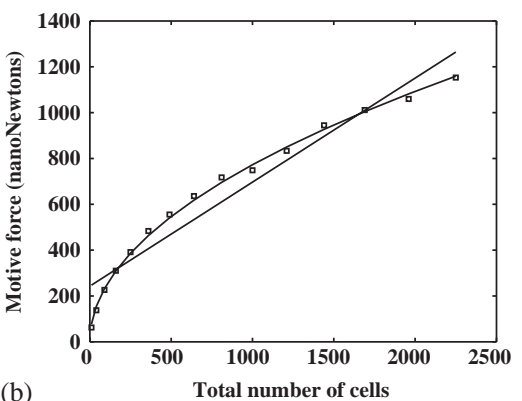

Fig. 4. The average motive force as a function of the number of the cells in contact with the substrate (a), and as function of the total number of cells in the slug (b). The boxes indicate data from the simulations, the straight lines are linear least-square fits to the data, and the curve in (b) is the least squares fit to a square root function. In all the simulations the slug starts out 10 cells long with square cross-sections that vary from 1 to 15 cells on a side. The cells are stimulated to move by a cAMP wave as described in the text. The motive force is the average motive force for the last 8 min of a 10 min simulation, done with a time-step of $0.002 \mathrm{~min}$.
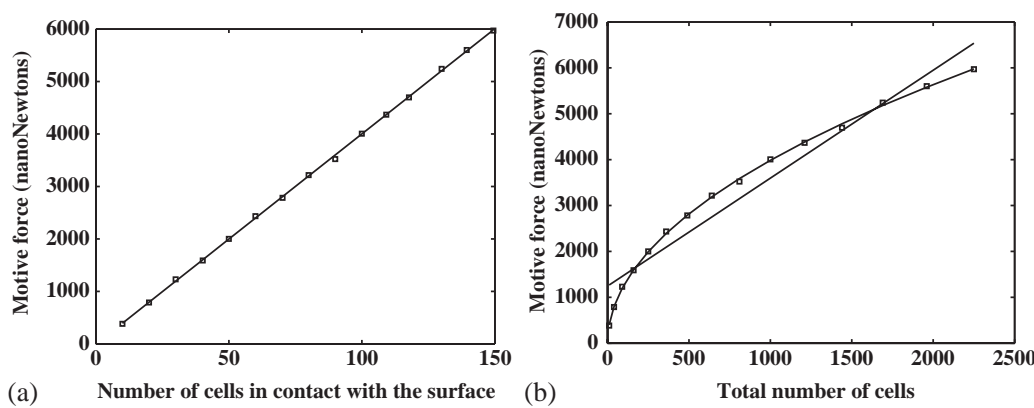

Fig. 5. The average motive force as a function of the number of cells in contact with the substrate (a), and as a function of the number of cells in the slug (b), when all cells are moving simultaneously. The figure is plotted in the same manner as Fig. 4 and the simulations use the same parameters. A table of the data is given in Appendix A.

approximations stem from the fact that the slug does not remain a precise rectangular solid during migration (cf. Fig. 3).

As might be expected, the same results hold when all cells are in motion, although in both sets of simulations the dimension of the cross-section of the slug must be larger than the length before the trend is clearly visible (see Fig. 5). To check that our results scale with the size of the slug, we ran a simulation for a slug $15 \times 15 \times 100$ cells, which is comparable to those used in experiments. The motion stimulus was also scaled to correspond with a cAMP wave with a wave speed of $300 \mu \mathrm{m} / \mathrm{min}$, a wave width of 200 and $1000 \mu \mathrm{m}$ from wave front to wave front. The average motive force per actively moving 
cell in contact with the substrate was $38.5 \mathrm{nN}$. This simulation suggests that the results are not dependent on the size of the slug.

The simulations results show that the motive force scales with the surface area and not with the volume, which is consistent with the theoretical analysis in Section 2 since the cells never 'freeze' during a movement cycle. Moreover, the overall motive force divided by the number of cells incontact with the substrate (the slope of the line plotted in Fig. 5) is approximately the same as the force any individual cell can generate when actively moving. We also calculated the time-averaged motive force for individual cells. For the simulation in which all cells are moving and the initial slug configuration is a $3 \times 3 \times 10$ cell slug, the motive force for a cell which is located half way down the length of the slug and is in contact with the substrate the entire simulation is $36.3 \mathrm{nN}$, whereas the motive force of the cell initially located just above it (in the center of the slug) is $0.58 \mathrm{nN}$. Thus cells not in contact with the substrate are not contributing to the translation of the center of mass of the slug.

To explore the possibility that a rigid framework may enable other cells to transmit force to the substrate, we first examined how the stiffness of the cells affects the results. If the cells are rigid enough, it might be possible for some force to be transferred to the surface by a cell not in contact with the surface through the rigid cells. Our simulations indicate that changing $k_{2}$ by a factor of 1000 does not alter the curves or their slopes. Presumably this is due to the relatively gradually buildup of forces, which allows time for the viscoelastic elements to adjust. Next we allowed a randomly chosen subset of cells to be stationary at any given time. In simulations in which every $6 \mathrm{~s} 25 \%$ of the cells froze, we again found that the forces scales with surface area (see Fig. 6). It is likely that with the small percentage of frozen cells there was not much of a network which actually connected to the substrate. As the percentage of cells which are frozen increases the slug tends to loose

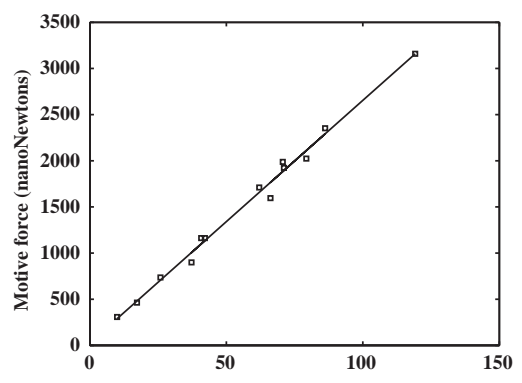

(a) Number of cells in contact with the surface

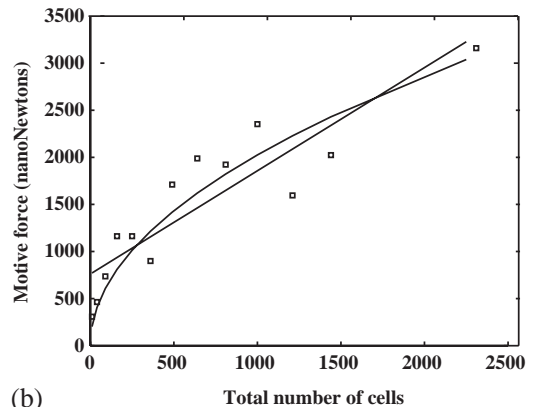

(b)

Fig. 6. The average motive force is plotted vs. the number of cells in contact with the substrate in (a) and vs. the total number of cells in the slug in (b) for simulations in which a portion of the cells are frozen. The data does not follow the square root curve because the length of the slugs during the course of a simulation changes dramatically in some cases. The data is plotted as in Fig. 5 and the simulation parameters are the same. The only difference is that at any time $25 \%$ of the cells are frozen for a duration of $6 \mathrm{~s}$ and the time step varied between 0.002 and 0.0005 min, depending on the simulation.
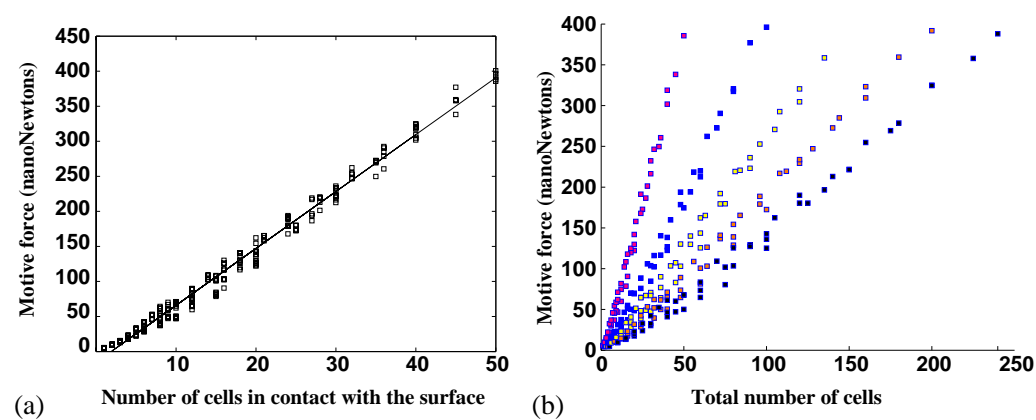

Fig. 7. Data from 250 different simulations are plotted with the vertical axis as the translational force of the slug in nanoNewtons and the horizontal axis as the number of cells in contact with the surface (a), or the total number of cells in the slug (b). Five lines are clear in (b): these correspond to subsets of the data in which the initial height of the slug is held constant, which implies that the volume of the slug and the surface area in contact with the substrate are proportional. All simulations started with a 3D array of cells whose dimensions ranged from 1 to 5 in both dimensions transverse to the length of the slug and 1 to 10 in length. The vertical axis is the average translational force for the last 8 min of a 10-min simulation, done with a time step of $0.002 \mathrm{~min}$. In all the simulations, cells are stimulated to move one direction by a traveling wave. Because the cells are not constantly moving the time averaged force for one cell should be about $8 \mathrm{nN}$ which is consistent with the data shown. 
cohesiveness. In addition if the frozen cells remain stationary for too long, the slug again breaks apart. We believe that this type of mechanism is unlikely in Dd due to the difficulties it creates in maintaining a cohesive slug, although we cannot rule out the possibility that a cAMP may synchronize freezing in such a way as to facilitate transmission of force from the interior.

Finally, we ran a set of simulations in which the number of cells in a slug varied independently in all three dimensions. This produces some configurations which are not at all like a slug, but it clearly illustrates the dependency of the force upon the contact surface area. The initial configurations ranged through 250 cases having from 1 to 5 cells along the width and height, and from 1 to 10 cells along the length of the slug. In all cases the cells were stimulated to move in a fixed direction by a traveling wave. The results are shown in Fig. 7.

\section{Discussion}

\subsection{The continuum approach}

Odell and Bonner (1986) derived a continuum fluid mechanical model for slug movement that incorporated assumptions about the behavior of individual amoebae. In their model active stresses were derived from an active relative velocity field set up at the cell surface. Each amoeba was thought of as a 'cortical tractor' pulling the surrounding viscous fluid past itself by rotating its membrane (at different rates on different parts of the surface). Although there are clearly two phases involved, some form of local averaging, which was not specified, lead to a model in which only a single phase appeared. The continuum was effectively an incompressible viscous fluid subject to the action of 'stirrers' embedded within it. The fluid (both within and outside the cells) was modeled as incompressible and the stress resulting from bulk flow of the single phase was hypothesized to be entirely due to viscous drag in response to gradients in the membrane velocity. This was the only way in which cells interacted in the model.

The authors show that there can be no net movement unless there is a chemokinesis chemical that controls the intensity of cell movement, and that the gradient of this chemical must be transverse to the axial gradient of cyclic AMP. While this is an important attempt at modeling the motion of the slug, there are several difficulties with the model. Firstly, it is known (Pauline Schaap, personal communication) that there is very little slime in the interior of the slug. Only anterior cells secrete it and it remains highly localized in the surrounding sheath. There may in fact be essentially no fluid in the interior, in which case the stirrer model cannot work. Moreover, traction forces on the sheath play no role in the model; in essence the cells swim in the extracellular matrix. However, the sheath must play an essential role in confining the cells in the pulsatile motion, for the slug lifts off the substrate, and it is difficult to understand how this can occur without interactions between the sheath and the cells within the slug.

More recently Umeda and Inouye (1999) have developed a continuum model that has certain features in common with the PO model and the extension we develop here. In this model migrating cells synthesize new membrane at the front and degrade it at the rear, and the stationary network allows transmission of stress to the substrate. The authors postulate a motive force that is proportional to the volume and solve the resulting continuum equations for the motion in two space dimensions. The model does produce the correct 2D geometry of the slug, the cell sorting, and a correlation between the speed of the slug and the length of the slug, but there is as yet little evidence that the postulated recycling of membrane occurs, and in fact, the scenario presented is at variance with the current understanding of how single cells move on a fixed substrate. Other continuum models developed by Vasiev et al. (1997), Bretschneider et al. (1999), and Vasiev and Weijer (2003) also model the slug as a viscous fluid which exerts force. Chemotactic movement of cells is controlled by cAMP waves, and it is postulated that the motive force is proportional to the volume. The active force is proportional to the local gradient of attractant, but how the macroscopic forces arise from the behavior of individual cells is not addressed.

The recent continuum models can replicate slug motion by suitable choices of the model parameters, but only the model due to Umeda and Inouye (1999) attempts to relate the force to a microscopic description of cell motion. Our computational results using a cellbased model suggest that any continuum description should have the property that the force exerted by the slug on the substrate should be solely due to the active force exerted by cells in contact with the substrate; interior cells contribute essentially nothing to the motion of the center of mass. However, the continuum models are based on experimental observations that suggest that force may be proportional to the volume, in contrast to the results of the simulations, and we address the discrepancy in the following section.

\subsection{The cell-based approach}

Our theoretical predictions show that there must be some mechanism that causes a subset of cells to "freeze" transiently in order for the cells not in contact with the substrate to contribute to the overall motive force of a slug. The computational experiments show that this did not work with two types of simulation, but there are 
other possibilities. In the first experiment the cells were stiffened uniformly, but still only the cells in contact with the substrate contributed to the overall motive force of the slug. The second type of simulation was to freeze a random subset of cells, but in this case it was difficult to maintain the integrity of the slug.

How can our results be reconciled with the experimental results which indicate that the motive force of the slug does scale with the volume (Inouye, 1984)? In order to answer this question, we first consider the possibility that there is some relationship between a slug's radius and its length, and second we examine some assumptions made in interpreting the experimental data.

One factor that may confound the interpretation of data is that in naturally developing slugs the radius and the length seem to be correlated. Data taken from Inouye and Takeuchi (1979) is plotted in Fig. 8, and one sees there that a linear relationship is very likely. The correlation coefficient between the length and the width of the slugs is 0.78 when the two longest slugs are included in the fit, and 0.93 when they are excluded. Also, in some experiments the slugs move in capillary holes made in the agar (Inouye and Takeuchi, 1980; Inouye, 1984), and these tunnels may impose some relationship on the length and the radius of the slugs, constricting the radius of large slugs and allowing smaller slugs to expand.

As is evident in Figs. 5 and 4, the plots of the motive force with respect to volume appear to be linear whenever the radius is less than the length. It is only when the radius exceeds the length that the true dependency is revealed. Since the length is fixed initially in our simulations, if we assume that the dependency of force is on contact surface area, a plot against volume should be, and indeed is, a square root function. In fact,

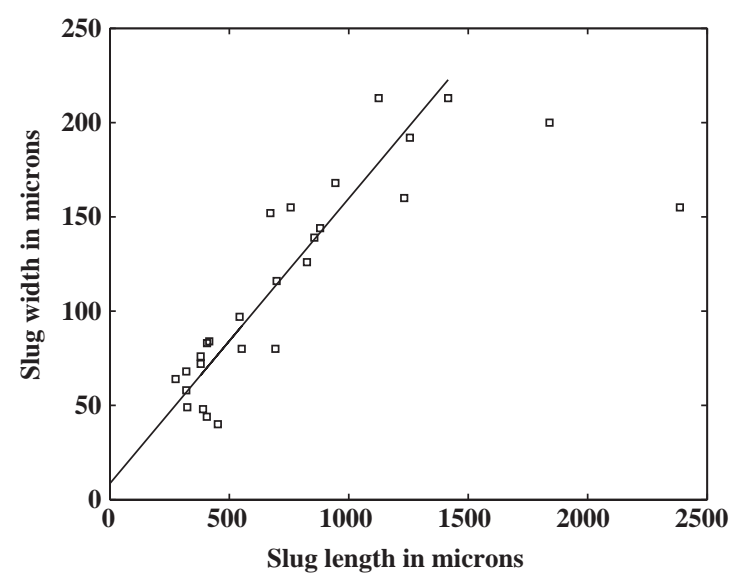

Fig. 8. The width of slugs is plotted against the length of the slug. The line is the least squares fit to the data, not including the two longest slug data points. The correlation coefficient is 0.93 when these two data points are excluded, and thus a linear relation is very likely (data is taken from Inouye and Takeuchi, 1979). one can easily conclude that the force scales with volume if only a subset of the data in Fig. 7 corresponding to aspect ratios ranging from 1:10 to $1: 1$ is plotted (cf. Fig. 9). The relationship of force to the surface area is only revealed when the complete data set is analysed.

Of course, if naturally occurring slugs always have a small radius when compared to the length of the slug, using a linear relationship could be a reasonable approximation to the true force relation. It may be possible to design experiments which eliminate this problem by measuring the motive force of slugs with much larger radii. Alternatively, by carefully controlling the size of the slugs one might be able to extract more information. For example, one could consider data for slugs of the same length but with different diameters, or consider slugs of the same volume with different diameters, by placing them in capillary tubes which restrict their diameter. In the second case, reducing the radius of the capillary by a factor of $v$ will only increase the surface area by a factor of approximately $v$, and unless the radii of the capillaries are varied over a large range, the surface areas may not change enough to give conclusive data.

Another problem may be in the assumptions used to interpret some of the data. In Inouye and Takeuchi (1979) the authors assume that the motive force for a slug migrating on agar is proportional to the volume and derive the relation

$\frac{f}{v^{*}}=\frac{s}{L}+a \equiv \delta(L)$,

where $f$ is the motive force per unit volume, $v^{*}$ is the speed, $L$ is the length of a slug, $s$ is a coefficient that reflects resistance at the ends of the slug, $a$ is a drag

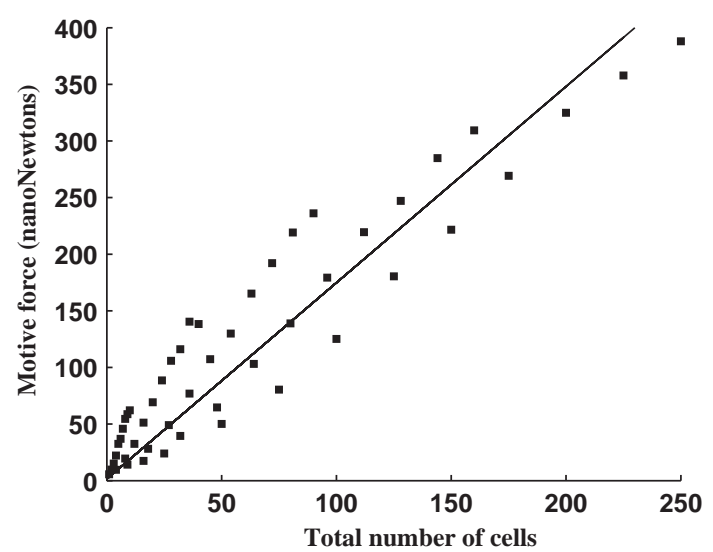

Fig. 9. This figure plots a subset of the data from Fig. 7 to illustrate that for some slug dimensions, the data can appear to have a linear relationship, only when a larger data set is considered can the true relationship be clearly seen (Fig. 7). Twenty five of the data points in Fig. 7 are replotted where the transverse cross-section of the slug is a square and the length of the slug ranges from 5 to 10 cells. The plotted line is the least squares fit to a line of the data and has a correlation coefficient of 0.97 . 
coefficient, and $\delta(L)$ is defined for later convenience. They then fit the velocity-length data for various slugs to the equation

$v^{*}=\frac{L}{\alpha+\beta L}$,

where $\alpha \sim 0.27, \beta \sim 0.26$. They thereby in effect obtain $s / f$ and $a / f$ and show that $s \sim a$.

In Inouye and Takeuchi (1980) the authors observe that the velocity of a slug migrating in an agar tube is always smaller than on an agar substrate, and from the regression line drawn in their Fig. 3 one can derive the relationship

$v=(0.25)(0.87)(4-L) v^{*}$.

Assuming that the force in the agar tube behaves according to Eq. (8), as was done in Inouye and Takeuchi (1980), it follows that the motive force is approximately given by

$$
\begin{aligned}
f & =\delta(L) v=\delta(L) \frac{v}{v^{*}} v^{*} \\
& =c_{1} \delta(L) \frac{L(4-L)}{1+L}=c_{2}(4-L),
\end{aligned}
$$

where $c_{1}$ and $c_{2}$ are constants of order 1 and we have set $s=a$ and $\alpha=\beta$. Thus the original assumption that the motive force is proportional to the volume turns out to be contradictory for the slugs moving in a tube.

There is another interpretation of the experimental data which also appears to conflict with our theoretical prediction. In Bonner (1995), Inouye's data is plotted with other data to show that the velocity of a slug is related to its length as $v \propto L^{0.66}$. If we assume that the slug is a half-cylinder and sum Eq. (2) over all cells, and further assume that the motive force is proportional to the surface area in contact with the substrate, we predict that the relationship should be

$v=\frac{k_{1} L}{k_{2} r+k_{3} L}$,

where $r$ is the radius of the slug and $k_{1}, k_{2}$, and $k_{3}$ are constants. If there is a linear relationship between $L$ and $r$ (see Fig. 8) or if $r \ll L$, the velocity is approximately constant which is consistent with our computational results. Although one could argue that the dependency $v \propto L^{0.66}$ is weak, it may be that other factors account for the discrepancy. In all of our calculations we assume that the same proportion of cells in the slug are actively moving, regardless of the slug size. However it is likely that different-sized slugs will have different signaling properties and thus different proportions of actively moving cells. In fact, in early experiments by Francis (quoted in Shaffer, 1964) the tip of a slug was replaced by a smaller one and the resulting slug moved more slowly, and larger tips grafted onto a slug resulted in faster migration. This might be due to the cAMP produced by the tip, which would cause a smaller or larger proportion of cells to move actively.

In conclusion, prior interpretations of experimental evidence appear to contradict the theoretical prediction that only cells in contact with the substrate contribute to the migration of the slug, but as we indicated above, there are several plausible reasons for this. The theoretical prediction is clear: without some special mechanism such as a transiently frozen subpopulation of cells, only those in contact with the substrate can gain traction to produce force which will translate the entire mass. We have not considered the slime sheath in this paper, but even if the entire surface area of the slug is effective for transmitting force, not just those cells in contact with the substrate as in our experiments, the motive force will still scale with the surface area of the slug and not its volume. Clearly more experiments are needed to definitively establish whether the total motive force scales with surface area or volume.

\section{Acknowledgements}

Research supported in part by NIH grant GM 29123, NSF grant DMS 9805494 and NSF grant DMS 0317372, the Max Planck Institute, Leipzig, and the Alexander von Humboldt Foundation.

\section{Appendix A}

In this appendix we first justify neglecting the acceleration term in the equations of motion (6) and the approximation used for the drag terms. Next the forces in the model are precisely defined and the details of how cells rotate is given. Finally we describe the numerical algorithm.

\section{A.1. Forces}

The Reynolds number is defined as

$R e=\frac{\rho \ell\|\mathbf{v}\|}{\mu}$,

where $\rho$ is the fluid density, $\ell$ is the characteristic length, $\mathbf{v}$ is a velocity vector, $\mu$ is viscosity and $\|\mathbf{v}\|$ is the standard norm in $\mathfrak{R}^{3}$. By letting $\|\mathbf{v}\|$ range from $5 \mu \mathrm{m} / \mathrm{h}$ to $30 \mu \mathrm{m} / \mathrm{min}$ (Bray, 1992), $\ell=10 \mu \mathrm{m}$ and using the density and viscosity of water we get a range for the Reynolds number of $1.7 \times 10^{-8}$ to $6.24 \times 10^{-6}$. For a viscosity more characteristic of cytoplasm the Reynolds number is still smaller.

In the PO model several simplifying assumptions about the drag are made and thus the drag, as is commonly done, is proportional to velocity. The drag 
due to the fluid acting on the $i$-th cell is simply given by

$\mathbf{D}_{f, i}=-6 \pi r_{i b} \mu_{f} \mathbf{v}_{i}$,

where $\mathbf{v}_{i}$ is the velocity of the $i$-th cell and $r_{i b}$ is the radius of the $\mathbf{b}$ axis of the $i$-th cell or $u_{b}+b_{0}$ (the drag equation is for a sphere not an ellipse). Since each cell can have a varying degree of contact with the fluid depending upon how many cells surround it, the drag is multiplied by the ratio of the surface area in contact with the fluid and the total surface area of the cell (see Eq. (6)). To simplify the computations the total surface area is approximated by the initial surface area.

\section{A.1.1. Adhesive forces}

Detailed modeling of cell adhesion is a major effort in itself, and as a simplification the PO model splits the adhesional force into two components, one tangential to the cell membrane and one normal to the membrane. This is motivated by the idea that cell adhesion may be less resistant to tangential forces and more resistant to normal forces (Keller et al., 2000).

The component of the adhesive force tangential to the cell membrane acts as a drag force opposing the motion of the cell. For the substrate adhesive force, Eq. (A.2) is used and again we multiply by the ratio of surface area in contact with the substrate and the total surface area. Thus the tangential adhesive force on the $i$-th cell due to adhesion with the substrate is

$\mathbf{D}_{0, i}=-6 \pi r_{i b} \mu_{s} \hat{\mathbf{v}}_{i}$

and the tangential adhesive force between cell $i$ and cell $j$ is

$\mathbf{D}_{j, i}=-6 \pi r_{i b} \mu_{\text {cell }}\left(\mathbf{v}_{i}-\mathbf{v}_{j}\right)$,

where $\mathbf{v}_{j}$ is the velocity of the $j$-th cell.

The normal forces due to adhesion and the rheological forces are treated as a potential energy well with the minimum energy occurring when the cell membranes slightly overlap. The overlap of membranes is interpreted as regions of the cell where there is local deformation of the cell shape away from the model's imposed ellipsoidal shape. In our version of the PO model we define the potential energy well differently. The attractive side of the potential well is similar in that the adhesion will draw the cells closer together. It is the repulsive side of the potential energy which is quite different. The repulsive force using the viscoelastic elements is calculated. We also assume cells to be slightly larger (1.2 times) than the viscoelastic elements. This means that there is a region around the cell where it does not resist compression and can form adhesive contacts with other cells. Thus if cell membranes are separated beyond this distance there is no adhesive force, if cell membranes overlap in this region the adhesive force is positive causing them to draw closer, and if the cells overlap beyond this region the viscoelastic properties of the cells usually cause the cells to push apart.

In order to be precise we define the following vectors. Let $\mathbf{d}_{j, i}$ be the vector from center of cell $i$ to its edge in the direction of the vector from the center of cell $i$ to the center of cell $j, \mathbf{h}_{j, i}$ (see Fig. 10). The vector $\mathbf{s}_{j, i}$ is from the edge of cell $i$ to the edge of cell $j$ in the direction of $\mathbf{h}_{j, i}$. The membranes of cell $i$ and cell $j$ overlap when $\hat{\mathbf{s}}_{j, i} \cdot \hat{\mathbf{h}}_{j, i}=-1$. Throughout Appendix A the caret notation indicates a unit vector.

For adhesion between cells the normal force is given by

$\mathbf{A}_{j, i}= \begin{cases}\alpha A_{i j} \hat{\mathbf{s}}_{j, i} & \text { if } \overbrace{\left\|\mathbf{s}_{j, i}\right\| \leqslant .2\left\|\mathbf{d}_{j, i}\right\|+.2\left\|\mathbf{d}_{i, j}\right\|}^{\text {cells within range }} \text { and } \overbrace{\hat{\mathbf{s}}_{j, i} \cdot \hat{\mathbf{h}}_{j, i}=1}^{\text {no overlap }} \\ 0 & \text { otherwise. }\end{cases}$

The coefficient $\alpha$ is the density of adhesion receptors per unit area multiplied by the force per receptor. The area of contact between the $i$ cell and the $j$ cell is denoted $A_{i j}$. We approximate this area by assuming the cells are spheres with radii $r_{i}=1.2\left\|\mathbf{d}_{j, i}\right\|$ and $r_{j}=1.2\left\|\mathbf{d}_{i, j}\right\|$. We take the radii of the two cells to be slightly larger than the actual cells. This accounts for the fact that the cells can reach out to nearby cells and have a tendency to make contact. The contact area is the area of the region bounded by the circle of intersection of the two spheres. Thus

$$
\begin{aligned}
& \begin{aligned}
A_{i j}= & \frac{\pi}{4\left\|\mathbf{h}_{j, i}\right\|^{2}}\left(2|| \mathbf{h}_{j, i} \|^{2}\left(r_{i}^{2}+r_{j}^{2}\right)+2 r_{i}^{2} r_{j}^{2}\right. \\
& \left.\quad-r_{i}^{4}-r_{j}^{4}-\left\|\mathbf{h}_{j, i}\right\|^{4}\right)
\end{aligned} \\
& \text { and } A_{i j}=A_{j i} \text {. So } \\
& \mathbf{A}_{i, j}=-\mathbf{A}_{j, i} .
\end{aligned}
$$

For computational convenience we multiply $\mathbf{A}_{j, i}$ by the following function evaluated at $\left\|\mathbf{s}_{j, i}\right\|$, to smooth the discontinuity in the forces

$f(x)= \begin{cases}\left|\sin \frac{\pi}{2} \frac{x}{2|| \mathbf{d}_{j, i}||}\right| & \text { for }-.2\left\|\mathbf{d}_{j, i}||<x<.2\right\| \mathbf{d}_{j, i} \|, \\ 1 & \text { otherwise. }\end{cases}$

We treat the normal adhesive force due to cell-substrate adhesion in a more realistic manner. In reality the adhesion force will counter any force up to a threshold which would separate the cells from the substrate and it
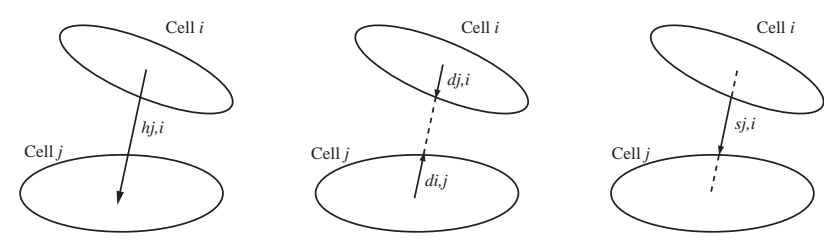

Fig. 10. The three sketches above show the vectors $\mathbf{h}_{j, i}, \mathbf{d}_{j, i}, \mathbf{d}_{i, j}$ and $\mathbf{s}_{j, i}$. 
will bring the cells to an optimal contact with the substrate. Thus for adhesion with the substrate, we have a potential energy function which brings the cell to an optimal distance from the substrate (the rheological forces form the other part of the potential energy well, pushing away from the substrate when the cell is too close) but we also counter any forces pulling the cell away from the substrate which are below a threshold. Specifically the normal force due to adhesion to the substrate is given by

$$
\mathbf{A}_{0, i}=\left\{\begin{array}{cc}
\left(-\mathbf{F}_{i} \cdot \mathbf{k}+\mathbf{A}_{0, i}^{p}\right) \mathbf{k} & \text { if } 0 \leqslant \mathbf{F}_{i} \cdot \mathbf{k} \leqslant S_{a d} \text { and } \\
& -.2\left\|\mathbf{d}_{0, i}\right\| \leqslant\left(\mathbf{s}_{0, i} \cdot-\mathbf{k}\right) \\
& \leqslant .2\left\|\mathbf{d}_{0, i}\right\|, \\
0 & \text { otherwise, }
\end{array}\right.
$$

where $S_{a d}$ is a positive constant representing the adhesion strength and $\mathbf{F}_{i}$ is the net force on the $i$-th cell given by

$$
\begin{aligned}
\mathbf{F}_{i}= & \left(\mathbf{M}_{0, i}+\mathbf{R}_{0, i}^{*}+\mathbf{A}_{0, i}+\sum_{j \neq i} \mathbf{T}_{j, i}+\sum_{j \neq i} \mathbf{A}_{j, i}\right. \\
& \left.+\sum_{j \neq i} \mathbf{R}_{j, i}+\sum_{j \neq i}\left(\mathbf{M}_{j, i}+\mathbf{R}_{j, i}^{*}\right)\right)
\end{aligned}
$$

and

$\mathbf{A}_{0, i}^{P}=-\sin \frac{\pi \mathbf{s}_{0, i} \cdot(-\mathbf{k})}{2} \frac{\mid \mathbf{d}_{0, i} \|}{,}$

where $\mathbf{d}_{0, i}$ is the vector from the center of the cell to its membrane in the $\mathbf{k}=(0,0,1)$ direction.

\section{A.1.2. Active forces}

The active force is the force which a cell generates through traction, tension or some other means, in order to move. In the PO model, cells "pull" in order to move. If a cell stimulated to move (for either low motility motion or high motility motion) is in contact with the substrate (a 2D plane), the component of the force in the plane of the substrate is obtained by "pulling" on the substrate. For the other component of the force, the $z$ component, or in the case where the cell is not in contact with the substrate, the entire force is generated by pulling on the neighboring cell whose center is closest to the line in the direction of desired motion. If there is no cell $90^{\circ}$ in any direction of the direction of motion, the cell cannot "grab" a cell and only exerts force on the substrate if it is close enough.

Before precisely defining the force on the substrate, we define the vector of the active force generated by cell $i$ to be $\mathbf{T}_{i}$ which is in the opposite direction of the desired motion. The force applied by the $i$-th cell on the substrate due to pulling is given by

$$
\mathbf{T}_{i, 0}= \begin{cases}0 & \text { if }\left\|\mathbf{s}_{0, i}\right\| \geqslant\left(\left\|\mathbf{d}_{j, i}\right\|+\left\|\mathbf{d}_{i, j}\right\|\right), \\ \mathbf{T}_{i} \cdot \mathbf{i}+\mathbf{T}_{i} \cdot \mathbf{j} & \text { otherwise }\end{cases}
$$

where $\mathbf{s}_{s, i}$ is the vector from the edge of cell $i$ 's membrane to the substrate in the $-\mathbf{k}$ direction, $\mathbf{i}=(1,0,0)$, and $\mathbf{j}=(0,1,0)$. This force does not move the substrate since it is assumed to be fixed, but associate with this force is an equal and oppose force, $\mathbf{M}_{0, i}$, which can affect the cell shape and position.

In order to define the force one cell applies to another, first the cell to which the force will be applied must be identified. A set of indices $\mathscr{N}_{i}^{a}$ identifies the neighboring cells of the $i$-th cell or the cells within a distance $d_{n}$, a positive constant, and $j$ is index for the cell whose center is in the closest direction to the direction of $\mathbf{T}_{i}$. Let $j$ be given by

$j=\left\{k: \max _{k \in \mathscr{N}_{i}^{a}} \frac{\mathbf{x}_{k}-\mathbf{x}_{i}}{\left\|\mathbf{x}_{k}-\mathbf{x}_{i}\right\|} \cdot \hat{\mathbf{T}}_{i}\right\}$,

where $\mathbf{x}_{n}$ is the location of the center of the $n$-th cell and $\mathscr{N}_{i}^{a}=\left\{k: 0<\left\|\mathbf{x}_{k}-\mathbf{x}_{i}\right\| \leqslant d_{n}\right\}$.

The force applied to the $j$-th cell by the $i$-th cell due to pulling is given by

$\mathbf{T}_{i, j}= \begin{cases}0 & \text { if } j=\emptyset, \\ \left(\mathbf{T}_{i} \cdot \mathbf{k}\right) \mathbf{k} & \text { if }\left\|\mathbf{s}_{0, i}\right\| \leqslant .2\left(\left\|\mathbf{d}_{j, i}\right\|+\left\|\mathbf{d}_{i, j}\right\|\right) \\ & \quad \text { and } j \neq \emptyset, \\ \mathbf{T}_{i} & \text { otherwise. }\end{cases}$

When an unstimulated cell is stimulated by the motion stimulus, it orients itself and generates active force in order to move in high motility motion for as long as it is being stimulated by the motion stimulus. In our simulations the active force is ramped up and down. In addition there is some random variation given to the force due to the random differences in cells. Not only is this biologically realistic but it is computationally simpler.

The forces and ramping for the active force are applied in the following manner:

$\left\|\mathbf{T}_{j, i}\right\|=r(t) r_{a} g_{f}$,

where $g_{f}$ is a parameter defining the magnitude of the active force, $r_{a}$ is a randomly generated number in the interval $[0.8,1.2]$ and

$r(t)= \begin{cases}t-t_{a} & \text { for } 0<t-t_{a}<t_{r}, \\ 1 & \text { for } t_{r} \leqslant t-t_{a} \leqslant t_{d}, \\ 1-\left(t-t_{d}\right) & \text { for } 0<t-t_{d} \leqslant t_{r},\end{cases}$

where $t_{r}$ is the time duration of the ramp, $t_{a}$ is the first time the cell is stimulated to move since not being stimulated and $t_{d}$ is the time the cell stops receiving the motion stimulus. If the cell is not being stimulated by the 
motion stimulus, it will either generate force for low motility motion which includes no force depending on the parameters used. For all simulations $t_{d}=20 \mathrm{~h}$ where $h$ is the time step. Forces are ramped down in a similar fashion when cells are no longer stimulated to move.

\section{A.1.3. Internal forces}

Two abutting cells may produce a local deformation of the membranes to accommodate the volume of each cell which would otherwise occupy the same space. This local compression is characterized by overlapping cell membranes (see Fig. 11), and when a cell is deformed at a point of contact, it will push or pull on neighboring cells depending on what the net forces acting on it. If a cell is stretched and relaxing and another cell is deforming it, the relaxing cell will pull the other cell towards it provided the adhesion is strong enough.

When cells overlap, we assume that one or both cells are being locally compressed. In our implementation, the cell with the smallest absolute value internal pressure, $\left|p_{k}\right|$ is the cell which deforms and is locally compressed the most. The value $p$ represents the change in internal pressure. If it is a large, positive value, the cell is turgid due to the internal fluid. If the value is large and negative, the cell is stiff due to the internal cytoskeleton. When the values are not large the true situation is more complicated and how easily deformable the cell is depends on the combined influences of the internal fluid and the state of the cytoskeleton i.e., if the viscoelastic element is expanding or contracting.

The force due to locally compressed cells is given by and

$d_{i}=0.5 \max _{\ell \in \mathscr{N}_{i}^{o}}|| \mathbf{s}_{\ell, i}||\left(1-\left|\frac{\left|\bar{p}_{i}\right|-|| \bar{p}_{\ell} \mid}{\max \left(|| \bar{p}_{i}|,|\left|\bar{p}_{\ell}\right|\right)}\right|\right)$.

The values $d_{i}$ and $d_{j}$ represent the amount cell $i$ and cell $j$ are deformed and their sum gives the total deformation $d_{i}+d_{j}=\left\|\mathbf{s}_{j, i}\right\|$. The values $q_{a}, q_{b}$, and $q_{c}$ are determined by the viscoelastic elements in the cells and $\mathscr{N}_{i}^{o}$ is the set of cell indices whose cell membranes overlap with the membrane of cell $i$.

To determine the force with which the cell resists compression we solve Eq. (3) for each cell assuming the cell shape changes from its current shape to a new shape where it has been compressed by $d_{i} \hat{\mathbf{s}}$ in time $\mathrm{d} t$. We resolve the vector $d_{i} \hat{\mathbf{s}}$ into the direction of the axis of the cell, thus $d_{i} \hat{\mathbf{s}}=\alpha_{i} \hat{\mathbf{a}}_{i}+\beta_{i} \hat{\mathbf{b}}_{i}+\gamma_{i} \hat{\mathbf{c}}_{i}$ where $\alpha_{i}=\left(d_{i} \hat{\mathbf{s}} \cdot \hat{\mathbf{a}}_{i}\right), \quad \beta_{i}=\left(d_{i} \hat{\mathbf{s}} \cdot \hat{\mathbf{b}}_{i}\right)$ and $\gamma_{i}=\left(d_{i} \hat{\mathbf{s}} \cdot \hat{\mathbf{c}}_{i}\right)$. Solving Eq. (3) using an integrating factor we get

$$
\begin{aligned}
q_{a}\left(d_{i} \hat{\mathbf{s}}, i\right)=- & f_{a}\left(t_{c}+\mathrm{d} t\right), \\
f_{a}\left(t_{c}+\mathrm{d} t\right)= & \mathrm{e}^{-\zeta \mathrm{d} t} f_{a}\left(t_{c}\right)+f_{2}\left(a-\left|\alpha_{i}\right|\right) \\
& -\mathrm{e}^{-\zeta(\mathrm{d} t)} f_{2}(a) \\
& -\left(1-\mathrm{e}^{-\zeta \mathrm{d} t}\right) \bar{p}+k_{1} \mathrm{e}^{-\zeta\left(t_{c}+\mathrm{d} t\right)} \\
& \times \int_{t_{c}}^{t_{c}+\mathrm{d} t} \mathrm{e}^{\zeta t} \frac{\mathrm{d} a}{\mathrm{~d} t} \mathrm{~d} t,
\end{aligned}
$$

where $\zeta=k_{a} / \mu_{a}, t_{c}$ is the current time and $a=a\left(t_{c}\right)$ and is the length of one of the axes.

$\mathbf{R}_{j, i}=\{\begin{array}{l}-\mathbf{G}\left(d_{i} \hat{\mathbf{s}}_{i, j}, i\right)+\mathbf{G}\left(d_{j} \hat{\mathbf{s}}_{j, i}, j\right) \\ 0\end{array} \overbrace{\begin{array}{c}\hat{\mathbf{s}}_{j, i} \cdot \hat{\mathbf{h}}_{j, i}=-1 \\ \text { otherwise, }\end{array}}^{\text {cells overlap }}$ and $\overbrace{-\mathbf{G}\left(d_{i} \hat{\mathbf{s}}_{i, j}, i\right)+\mathbf{G}\left(d_{j} \hat{\mathbf{s}}_{j, i}, j\right) \leqslant\left\|\mathbf{A}_{j, i}\right\|}^{\begin{array}{c}\text { body forces do not pull more } \\ \text { than adhesive forces }\end{array}}$,

where

$$
\mathbf{G}(d \hat{\mathbf{s}}, i)=q_{a}(d \hat{\mathbf{s}}, i) \hat{\mathbf{a}}_{i}+q_{b}(d \hat{\mathbf{s}}, i) \hat{\mathbf{b}}_{i}+q_{c}(d \hat{\mathbf{s}}, i) \hat{\mathbf{c}}_{i}
$$

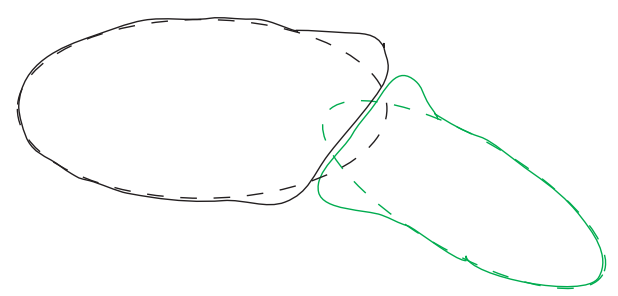

Fig. 11. The figure shows two cells whose membranes are overlapping. The dashed lines represent the ellipsoidal shape of the cells as represented in the model. The solid lines more accurately represent how the cells would locally deform. The cell with the pressure which is smallest in magnitude will deform the most.
Compression against the substrate works in the same manner with

$$
\mathbf{R}_{s, i}= \begin{cases}0 & \text { if } d_{i}>0 \\ -\mathbf{G}\left(d_{i} \mathbf{z}_{i}, i\right) & \text { otherwise }\end{cases}
$$

where $d_{i}$ is the distance from cell $i$ to the substrate in the direction of $\mathbf{z}_{i}=\left(0,0,-z_{i}\right)$.

\section{A.1.4. Cell rotation}

In the PO model, cells are oriented ellipsoids but here the details of how and when a cell changes its orientation are different. When a cell is engaged in directed motion it is assumed that two stimuli are provided, an orientation stimulus and a motion stimulus. The orientation stimulus provides a direction of orientation $\hat{\mathbf{d}}_{o}$, and the motion stimulus 
indicates if the cell should be engaged in directed motion.

Consider cell $i$ orientated in the direction of $\hat{\mathbf{a}}_{i}$, one of its major axes. When cell $i$ moves in high motility motion it does so in the direction of $\hat{\mathbf{a}}_{i}$. When an orientation cycle starts there are three possibilities. First, the cell is already oriented in the direction of the direction stimulus, $\hat{\mathbf{a}}_{i}=\hat{\mathbf{d}}_{o}$, in which there is no need to change orientation. Second, the cell is oriented in the opposite direction of the stimulus, $\hat{\mathbf{a}}_{i}=-\hat{\mathbf{d}}_{o}$. Experimentally it is observed that a cell either changes its leading edge to the opposite side and reverse its direction or the cell makes a " $U$ " turn maintaining the same leading edge. In our model we only allow the cell to change its leading edge by switching its orientation. Finally, if the orientation of the cell and the direction stimulus are not parallel, the cell changes its direction. Again it is experimentally observed that when a cell has established a leading edge, depending on the circumstances it can change directions by extending more pseudopods in one half of the leading edge causing the cell to veer in that direction. Consider a cell surrounded by other cells, in order to extend a pseudopod it may have to push a neighboring cell out of the way. In our model we approximate this by gradually rotating the cell about the vector $\hat{\mathbf{v}}=\left(v_{1}, v_{2}, v_{3}\right)=\hat{\mathbf{a}}_{i} \times \hat{\mathbf{d}}_{o} /\left\|\hat{\mathbf{a}}_{i} \times \hat{\mathbf{d}}_{o}\right\|$ until, upon completion of the orientation cycle, the cell orientation is the same as $\hat{\mathbf{d}}_{o}$. Before explaining why this is a good representation of the experimental observations, we first observe that as a simplification, in our model we do not take into account torque. When a cell pulls on another cell it will have multiple contacts with the other cell spreading the force over a segment of the membrane. Where the center of mass of the cell is with respect to the segment of the membrane will determine the strength of the torque. Because of this simplification, the rotation of the cell is equivalent to the cell retracting in the old direction and extending in the new direction. The neighboring cells are pushed as if the cell was extending a pseudopod. Of course, depending on the other forces, the cell may not be able to extend the pseudopod and may even retract in that direction.

When a cell changes direction via an orientation cycle, it may rotate until it is oriented in the new direction. Specifically in our model, the cell is rotated by a rotation matrix.

\section{A.2. Numerical methods}

\section{Our system is}

$\dot{\mathbf{x}}=\mathbf{M}^{-1}(\mathbf{x}) \mathbf{b}(\mathbf{x}, t)$,

where $\mathbf{M}$ is a $3 N \times 3 N$ real matrix, $\mathbf{v}=\dot{\mathbf{x}}$ and $\mathbf{b}$ are vectors with $3 N$ real components and $N$ is the total number of cells.
The entries of $\mathbf{M}$ and $\mathbf{b}$ are defined in terms of an intermediate function, the radii of the cells. Let $\mathbf{r}=$ $\left(\mathbf{r}_{1}, \mathbf{r}_{2}, \ldots, \mathbf{r}_{N}\right)$ where $\mathbf{r}_{i}=\left(r_{a_{i}}, r_{b_{i}}, r_{c_{i}}\right)$ is the vector of the radii for the $i$ cell and let $\mathbf{x}=\left(\mathbf{x}_{1}, \mathbf{x}_{2}, \ldots, \mathbf{x}_{N}\right)$ where $\mathbf{x}_{i}$ is the location of the center of the cell $i$. Therefore, the matrix $\mathbf{M}$ depends on $\mathbf{x}$ and $\mathbf{r}$, whereas $\mathbf{b}$ depends on $\mathbf{x}, \mathbf{r}$ and $t$. The difficulty arises since $\mathbf{r}$ also depends on $\mathbf{b}$. Thus we have a recursive definition for $\mathbf{r}$ with $\mathbf{r}(\mathbf{b}[\mathbf{r}, \mathbf{x}, t])$.

The quasi-implicit method is described in the following manner. Initially we discretize using the trapezoidal rule:

$$
\begin{aligned}
\mathbf{x}^{n+1}-\mathbf{x}^{n}= & \frac{h}{2}\left[\mathbf{M}^{-1}\left(\mathbf{r}, \mathbf{x}^{n+1}\right) \mathbf{b}\left(\mathbf{r}, \mathbf{x}^{n+1}, t_{n+1}\right)\right. \\
& \left.+\mathbf{M}^{-1}\left(\mathbf{r}, \mathbf{x}^{n}\right) \mathbf{b}\left(\mathbf{r}, \mathbf{x}^{n}, t_{n}\right)\right],
\end{aligned}
$$

where $\mathbf{x}^{n}=\mathbf{x}\left(t_{n}\right), t_{n}=h n$ and $h$ is the time step. We formulate a new problem so at each time step we can use the iterative nonlinear solver NKSOL (Brown and Saad, 1987) (a software package). Let $\mathbf{g}$ be defined by

$$
\begin{aligned}
\mathbf{g}(\mathbf{y})= & \mathbf{y}-\mathbf{x}^{n}-\frac{h}{2}\left[\mathbf{M}^{-1}(\mathbf{r}, \mathbf{y}) \mathbf{b}\left(\mathbf{r}, \mathbf{y}, t_{n+1}\right)\right. \\
& \left.+\mathbf{M}^{-1}\left(\mathbf{r}, \mathbf{x}^{n}\right) \mathbf{b}\left(\mathbf{r}, \mathbf{x}^{n}, t_{n}\right)\right] .
\end{aligned}
$$

Note that $\mathbf{g}\left(\mathbf{x}^{n+1}\right)=\mathbf{0}$. The cell radii represented in $\mathbf{r}$ are difficult to express as a function of $\mathbf{y}$ and $t$. Yet it is possible to determine $\mathbf{r}$ if $\mathbf{b}$ is given. Thus it would be simple to lag $\mathbf{r}$ in time and have a "quasi-implicit" scheme, but numerical tests indicate that this is not very efficient due to stability problems. Thus we use the most current information possible to calculate r. For a fixed time step $n$ we approximate $\mathbf{g}\left(\mathbf{y}^{i}\right)$ for each iteration by first finding $\mathbf{r}$, evaluating $\mathbf{g}$, and updating $\mathbf{r}$ in the following manner:

$$
\begin{aligned}
\mathbf{r}^{n+1, i,-}= & \mathbf{r}\left[\mathbf{b}\left(\mathbf{r}^{n+1, i-1,+}, \mathbf{y}^{i}, t_{n+1}\right)\right], \\
\mathbf{g}\left(\mathbf{y}^{i}\right) \approx & \mathbf{y}^{i}-\mathbf{x}^{n}-\frac{h}{2}\left[\mathbf{M}^{-1}\left(\mathbf{r}^{n+1, i,-}, \mathbf{y}^{i}\right)\right. \\
& \times \mathbf{b}\left(\mathbf{r}^{n+1, i,-}, \mathbf{y}^{i}, t_{n+1}\right) \\
& \left.+\mathbf{M}^{-1}\left(\mathbf{r}^{n}, \mathbf{x}^{n}\right) \mathbf{b}\left(\mathbf{r}^{n}, \mathbf{x}^{n}, t_{n}\right)\right], \\
\mathbf{r}^{n+1, i,+}= & \mathbf{r}\left[\mathbf{b}\left(\mathbf{r}^{n+1, i,-}, \mathbf{y}^{i}, t_{n+1}\right)\right] .
\end{aligned}
$$

To start the process $\mathbf{y}^{0}=\mathbf{x}^{n}, \mathbf{r}^{n+1,0,-}=\mathbf{r}\left(\mathbf{b}\left(\mathbf{r}^{n}, \mathbf{x}^{n}, t_{n+1}\right)\right.$ and $i=0,1, \ldots$.

\section{A.3. Parameters and data}

To determine the parameters for the viscoelastic elements we examined the creep function and the relaxation function of a standard linear solid. The creep function is the time evolution of the length of the element and the relaxation function is the time evolution of the force the element exerts. Fig. 12 shows these functions with the standard parameters used for the viscoelastic elements. If the cell is acted on by 10 other cells (a spherical, interior cell in our simulation can have 

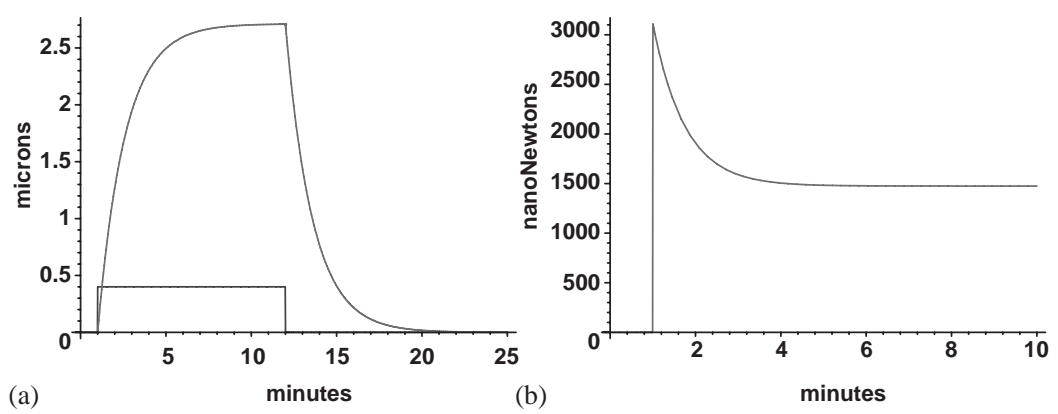

Fig. 12. The creep function for a standard linear solid and the applied force are plotted in (a) and the relaxation function is plotted in (b) with parameters $k_{1}=163.8 \mathrm{dyn} / \mathrm{cm}, k_{2}=147.5 \mathrm{dyn} / \mathrm{cm}$, and $\mu_{a}=123 \mathrm{dyn} \mathrm{min} / \mathrm{cm}$. The thin black line in (a) is a scaled plot of the applied force which starts at time 1 with a magnitude of $400 \mathrm{nN}$ and ends at time 12. The thicker gray line in plot (a) is the change in the length of the element (the diameter of the cell). In plot (b) the length of the element (diameter of the cell) at time 1 is fixed at $20 \mu \mathrm{m}$ (twice its resting length). The graph shows the force with which the element resists the deformation.

26 neighboring cells in contact), it will deform $2.5 \mu \mathrm{m}$ in $4 \mathrm{~min}$ and will return to it original shape about $7 \mathrm{~min}$ after the force is removed. The relaxation function shows that if a cell is stretched to double its length then it will initially exert $3000 \mathrm{nN}$ of force. These parameter values which may seem on the low end compared to measured values (see 2) still make the cells quite stiff.

Unless otherwise specified the parameters used in the simulations are $g_{a}=4 \times 10^{-} 3 \mathrm{dyn}, S_{a d}=0.1 \mathrm{dyn}, t_{c}=$ $0.33 \mathrm{~min}$ and $\alpha=2.414 \times 10^{6} \mathrm{nN} / \mathrm{mm}^{2}$. The initial cell size is a sphere of $5 \mu \mathrm{m}$ radius thus $a_{0}=b_{0}=c_{0}=5 \mu \mathrm{m}$.

\section{References}

Alberts, B., Johnson, A., Lewis, J., Raff, M., Roberts, K., Walter, P., 2002. Molecular Biology of the Cell, fourth ed. Garland, New York, London.

Alt, W., Dembo, M., 1999. Cytoplasm dynamics and cell motion: twophase flow models. Math. Biosci. 156 (1), 207-228.

Beniot, M., Gabriel, D., Gerisch, G., Gaub, H., 2000. Discrete interactions in cell adhesion measured by single-molecule force spectroscopy. Nat. Cell Biol. 2, 313-317.

Bonner, J.T., 1995. Why does slug length correlate with speed during migration in Dictyostelium discoideum?. J. Biosci. 20 (1), 1-6.

Bonner, J.T., 1998. A way of following individual cells in the migrating slugs of Dictyostelium discoideum. Proc. Natl Acad. Sci. USA 95 (Aug.), 9355-9359.

Bray, D., 1992. Cell Movements. Garland, New York.

Bretschneider, T., Vasiev, B., Weijer, C.J., 1997. A model for cell movement during dictyostelium mound formation. J. Theor. Biol. 189 (1), 45-51.

Bretschneider, T., Vasiev, B., Weijer, C.J., 1999. A model for dictyostelium slug movement. J. Theor. Biol. 199 (2), 125-136.

Brown, P.N., Saad, Y., 1987 (November). Hybrid krylov methods for non-linear systems of equations. Technical report ucrl-97645. Lawrence Livermore National Laboratory.

Caille, N., Thoumine, O., Tardy, Y., Meister, J.-J., 2002. Contribution of the nucleus to the mechanical properties of endothelial cells. J. Biomech. 25, 177-197.

Chung, C.Y., Funamoto, S., Firtel, R.A., 2001. Signaling pathways controlling cell polarity and chemotaxis. Trends Biochem. Sci. 26 (9), 557-566 (Review).
Dallon, J.C., Othmer, H.G., 1997. A discrete cell model with adaptive signalling for aggregation of Dictyostelium discoideum. Philos. Trans. Roy. Soc. London: Series B 352 (1357), 391-417.

Dallon, J.C., Othmer, H.G., 1998. A continuum analysis of the chemotactic signal seen by Dictyostelium discoideum. J. Theor. Biol. 194, 461-483.

Dembo, M., Wang, Y.-L., 1999. Stresses at the cell-to-substrate interface during locomotion of fibroblasts. Biophys. J. 76, 2307-2316.

DiMilla, P.A., Barbee, K., Lauffenburger, D.A., 1991. Mathematical model for the effects of adhesion and mechanics on cell migration speed. Biophys. J. 60 (1), 15-37.

Dong, C., Skalak, R., 1992. Leukocyte deformability: finite element modeling of large viscoelastic deformation. J. Theor. Biol. 158 (2), 173-193.

Feneberg, W., Westphal, M., 2001. Dictyostelium cells' cytoplasm as an active viscoplastic body. Eur. Biophys. J. 30, 284-294.

Fey, P., Stephens, S., Titus, M.A., Chisholm, R.L., 2002. SadA, a novel adhesion receptor in Dictyostelium. J. Cell Biol. 159 (6), 1109-1119.

Futrelle, R., Traut, J., McKee, W.G., 1982. Cell behavior in Dictyostelium discoideum: preaggregation response to localized cyclic AMP pulses. J. Cell Biol. 92, 807-821.

Galle, J., Loeffler, M., Drasdo, D., 2004. Modelling the effect of deregulated proliferation and apoptosis on the growth dynamics of epithelial cell populations in vitro. Biophys. J., submitted for publication.

Gracheva, M.E., Othmer, H.G., 2003. A continuum model of motility in amoeboid cells. Bull. Math. Biol. 66, 167-193.

Harris, A.K., Wild, P., Stopak, D., 1980. Silicone rubber substrata: a new wrinkle in the study of cell locomotion. Science 208, 177-179.

Harris, T.J.C., Ravandi, A., Siu, C.-H., 2001. Assembly of glycoprotein- 80 adhesion complexes in dictyostelium. J. Biol. Chem. 276 (52), 48764-48774.

Inouye, K., 1984. Measurement of the motive force of the migrating slug of Dictyostelium discoideum by a centrifuge method. Protoplasma 121, 171-177.

Inouye, K., Takeuchi, I., 1979. Analytical studies on migrating movement of the pseudoplasmodium of Dictyostelium discoideum. Protoplasma 99, 289-304.

Inouye, K., Takeuchi, I., 1980. Motive force of the migrating pseudoplasmodium of the cellular slime mould Dictyostelium discoideum. J. Cell Sci. 41, 53-64.

Janmey, P.A., 1991. Mechanical properties of cytoskeletal polymers. Curr. Opin. Cell Biol. 2, 4-11. 
Keller, R., Davidson, L., Edlund, A., Elul, T., Exin, M., Shook, D., Skoglund, P., 2000. Mechanisms of convergence and extension by cell intercalation. Philos. Trans. R. Soc. London B 355, 897-922.

Lo, C.M., Wang, H.B., Dembo, M., Wang, Y.L., 2000. Cell movement is guided by the rigidity of the substrate. Biophys. J. 79 (1), 144-152.

MacKintosh, F.C., 1998. Theoretical models of viscoelasticity of actin solutions and the actin cortex. Biol. Bull. 194, 351-353.

Maree, A.F., Hogeweg, P., 2002. Modelling dictyostelium discoideum morphogenesis: the culmination. Bull. Math. Biol. 64 (2), 327-353.

Mitchison, T.J., Carmer, L.P., 1996. Actin-based cell motility and cell locomotion. Cell 84 (3), 371-379.

Mogilner, A., Verzi, D.W., 2002. A simple nematode sperm physical model for the crawling 1-D cell. J. Stat. Phys. 110, 1169-1198.

Mogilner, A., Marlang, E., Bottino, D., 2000. A minimal model of locomotion applied to the steady gliding movement of fish keratocyte cells. In: Maini, P., Othmer, H. (Eds.), Mathematical Models for Biological Pattern Formation. Springer, New York, pp. 269-294.

Odell, G.M., Bonner, J.T., 1986. How the Dictyostelium discoideum grex crawls. Philos. Trans. R. Soc. London 312, 487-525.

Oliver, T., Dembo, M., Jacobson, K., 1999. Separation of propulsive and adhesive traction stresses in locomoting keratocytes. J. Cell Biol. 145 (3), 589-604.

Palsson, E., Othmer, H.G., 2000. A model for individual and collective cell movement in Dictyostelium discoideum. Proc. Natl Acad. Sci. USA 97, 10448-10453.

Pate, E., Othmer, H.G., 1986. Differentiation, cell sorting and proportion regulation in the slug stage of Dictyostelium discoideum. J. Theor. Biol. 118, 301-319.

Radhakrishnan, K., Hindmarsh, A.C., 1994 (March). Description and use of LSODE, the livermore solver for ordinary differential equations. Technical report UCRL-ID-113855. Lawrence Livermore National Laboratory.

Sahai, E., Marshall, C.J., 2003. Differing modes of tumour cell invasion have distinct requirements for rho/rock signalling and extracellular proteolysis. Nat. Cell Biol. 8, 711-719.

Savill, N., Hogeweg, P., 1997. Modelling morphogenesis: from single cells to crawling slugs. J. Theor. Biol. 184, 229-235.

Schmid-Schönbein, G.W., Sung, K.-l.P., Tozeren, H., Skalak, R., Chien, S., 1981. Passive mechanical properties of human leukocytes. Biophys. J. 36, 243-256.

Schimd-Schönbein, G.W., Kosawada, T., Skalak, R., Chien, S., 1995. Membrane model of endothelial cells and leukocytes. A proposal for the origin of a cortical stress. AMSE J. Biomech. Eng. 117 (May), 171-178.

Shaffer, B.M., 1964. The acrasina. Adv. Morphogenesis 3, 301-322.
Sheetz, M.P., Felsenfeld, D., Galbraith, C.G., Choquet, D., 1999. Cell migration as a five-sep cycle. Biochem. Soc. Symp. 65, 233-243.

Skalak, R., Dong, C., Zhu, C., 1990. Passive deformations and active motions of leukocytes. AMSE J. Biomech. Eng. 112 (3), 295-302 (Review)

Small, J.V., 1989. Microfilament-based motility in non-muscle cells. Curr. Opin. Cell Biol. 1, 75-79.

Soll, D.R., 1995. International Review of Cytology, vol. 163. Academic Press, New York (Chapter The use of computers in understanding how animal cells crawl, pp. 43-104).

Sung, K.-L.P., Dong, C., Schmid-Schonbein, G.W., Chien, S., Skalak, R., 1988. Leukocyte relaxation properties. Biophys. J. 54, 331-336.

Tang, Y., Othmer, H., 1995. Excitation, oscillations and wave propagation in G-protein-based model of signal transduction in Dictyostelium discoideum. Philos. Trans. Roy. Soc. London B 349, 179-195.

Tang, Y., Othmer, H.G., 1994. A G-protein-based model of adaptation in Dictyostelium discoideum. Math. Biosci. 120 (1), 25-76.

Umeda, T., 1993. A thermodynamical model of cell distributions in the slug of cellular slime mold. Bull. Math. Biol. 55 (2), 451-464.

Umeda, T., Inouye, K., 1999. Theoretical model for morphogenesis and cell sorting in dictyostelium discoideum. Physica D 129, 189-200.

Umeda, T., Inouye, K., 2002. Possible role of contact following in the generation of coherent motion of dictyostelium cells. J. Theor. Biol. 219 (3), 301-308.

Usami, S., Wung, S.-1., Skierczynski, B.A., Skalak, R., Chien, S., 1992. Locomotion forces generated by a polymorphonuclear leukocyte. Biophys. J. 63, 1663-1666.

Vasiev, B., Weijer, C.J., 2003. Modelling of dictyostelium discoideum slug migration. J. Theor. Biol. 223, 347-359.

Vasiev, B., Siegert, F., Weijer, C.J., 1997. A hydrodynamic model for Dictyostelium discoideum mound formation. J. Theor. Biol. 184, $441-450$.

Ward, K.A., Li, W.-I., 1991. Viscoelastic properties of transformed cells: role in tumor cell progression and metastasis formation. Biorheology 28 (A65), 301-313.

Wolf, K., Mazo, I., Leung, H., Engelke, K., von Andrian, U.H., Deryugina, E.I., Strongin, A.Y., Brcker, E.-B., Friedl, P., 2003. Compensation mechanism in tumor cell migration: mesenchymalamoeboid transition after blocking of pericellular proteolysis. J. Cell Biol. 160 (2), 267-277.

$\mathrm{Xu}$, J., Tseng, Y., Wirtz, D., 2000. Strain hardening of actin filament networks. J. Biol. Chem. 46, 35886-35892.

Yeung, A., Evans, E., 1989. Cortical shell-liquid core model for passive flow of liquid-like spherical cells into micropipets. Biophys. J. 56 (1), 139-149. 